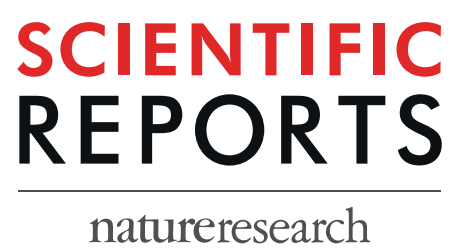

There are amendments to this paper

\title{
Onecut-dependent Nkx6.2
} transcription factor expression is required for proper formation and activity of spinal locomotor circuits

Mathilde Toch ${ }^{1}$, Audrey Harris ${ }^{1}$, Olivier Schakman $\mathbb{C}^{2}$, Elena Kondratskaya ${ }^{3}$, Jean-Luc Boulland $\mathbb{C}^{4}$, Nicolas Dauguet ${ }^{5}$, Stéphanie Debrulle ${ }^{1}$, Charlotte Baudouin ${ }^{1}$, Maria Hidalgo-Figueroa ${ }^{1,9,10}$, Xiuqian $\mathrm{Mu}^{6}$, Alexander Gow $\mathbb{1}^{7}$, Joel C. Glover ${ }^{3,4}$, Fadel Tissir $\mathbb{1}^{8}$ \& Frédéric Clotman ${ }^{1 *}$

In the developing spinal cord, Onecut transcription factors control the diversification of motor neurons into distinct neuronal subsets by ensuring the maintenance of $I s l 1$ expression during differentiation. However, other genes downstream of the Onecut proteins and involved in motor neuron diversification have remained unidentified. In the present study, we generated conditional mutant embryos carrying specific inactivation of Onecut genes in the developing motor neurons, performed RNA-sequencing to identify factors downstream of Onecut proteins in this neuron population, and employed additional transgenic mouse models to assess the role of one specific Onecut-downstream target, the transcription factor Nkx6.2. Nkx6.2 expression was up-regulated in Onecut-deficient motor neurons, but strongly downregulated in Onecut-deficient V2a interneurons, indicating an opposite regulation of $N k x 6.2$ by Onecut factors in distinct spinal neuron populations. $N k \times 6.2$-null embryos, neonates and adult mice exhibited alterations of locomotor pattern and spinal locomotor network activity, likely resulting from defective survival of a subset of limb-innervating motor neurons and abnormal migration of $\mathrm{V} 2 \mathrm{a}$ interneurons. Taken together, our results indicate that $\mathrm{Nkx6.2}$ regulates the development of spinal neuronal populations and the formation of the spinal locomotor circuits downstream of the Onecut transcription factors.

Movement is the principle expressive output of the central nervous system. Locomotor movements are initiated in the brain either cortically or subcortically, but are expressed by channeling activity to locomotor networks, located primarily in the ventral part of the spinal cord, that include rhythm-generating circuits called Central Pattern Generators (CPGs). The spinal locomotor networks ensure the integration of the multiple inputs that regulate motor activity and control the rhythm, speed, and coordination of the locomotor movements. CPGs are composed of interconnected spinal interneurons (INs) of diverse types that generate patterned activity that is then imposed on the motor neurons (MNs) that innervate skeletal muscles. Although many regulatory genes that direct the differentiation of these spinal neuron populations have been identified and the role of some of these genes has been extensively studied ${ }^{1}$, the complete genetic programs underlying the specification of the various neuron types within locomotor networks are far from fully deciphered.

\footnotetext{
${ }^{1}$ Université catholique de Louvain, Institute of Neuroscience, Laboratory of Neural Differentiation, Brussels, Belgium. ${ }^{2}$ Université catholique de Louvain, Institute of Neuroscience, Laboratory of Cell Physiology, Brussels, Belgium. ${ }^{3}$ Laboratory for Neural Development and Optical Recording (NDEVOR), Section for Physiology, Department of Molecular Medicine, Institute of Basic Medical Sciences, University of Oslo, Oslo, Norway. ${ }^{4}$ Norwegian Center for Stem Cell Research, Department of Immunology and Transfusion Medicine, Oslo University Hospital, Oslo, Norway. ${ }^{5}$ Université catholique de Louvain, de Duve Institute, Flow cytometry and cell sorting facility (CYTF), Brussels, Belgium. ${ }^{6}$ Department of Ophthalmology/Ross Eye Institute, New York State Center of Excellence in Bioinformatics and Life Sciences, University at Buffalo, Buffalo, NY, 14203, USA. ${ }^{7}$ Wayne state University, Center for Molecular Medicine and Genetics, Carman and Ann Adams Department of Pediatrics, Department of Neurology, Detroit, Michigan, USA. ${ }^{8}$ Université catholique de Louvain, Institute of Neuroscience, Laboratory of Developmental Neurobiology, Brussels, Belgium. ${ }^{9} \mathrm{CIBER}$ de Salud Mental (CIBERSAM), Madrid, Spain. ${ }^{10}$ University of Cadiz, Cadiz, Spain. *email: frederic.clotman@uclouvain.be
} 
During embryonic development, spinal MNs diversify into different subsets characterized by distinct molecular identities, locations, and synaptic connections. At the thoracic level, visceral MNs that innervate sympathetic neurons of the paravertebral ganglia are clustered in the preganglionic column (PGC) whereas somatic MNs that innervate dorsal axial muscles or body wall muscles are clustered in the median motor column (MMC) and the hypaxial motor column (HMC), respectively. At brachial and lumbar levels, MNs that innervate the ventral or dorsal regions of the limbs are clustered in the medial and lateral portions of the lateral motor column (LMCm and $\mathrm{LMCl}$ ), respectively ${ }^{2}$. LMC MNs further diversify into motor pools, each innervating a single mus$\mathrm{cle}^{3,4}$. MN diversification is controlled by a dynamic network of transcriptional regulators that include Onecut (OC) factors. OC factors, namely OC-1 (also called HNF-6 for Hepatocyte Nuclear Factor-6), OC-2 and OC-3, are transcriptional activators present in the digestive tract and the CNS during development ${ }^{5-9}$. In neural tissue, they regulate the production ${ }^{10}$, diversification ${ }^{11-13}$, distribution ${ }^{10,12-15}$ or maintenance ${ }^{10,15,16}$ of specific neuronal populations as well as the assembly of neuromuscular junctions ${ }^{17}$. In the spinal cord, OC factors are expressed in newly born $\mathrm{MNs}^{18}$, and control the diversification of MNs by directly regulating the expression of the Isl 1 gene $\mathrm{e}^{11}$. Furthermore, OC factors contribute to the progression of MN differentiation by recruiting MN-promoting transcriptional complexes to specific enhancer elements ${ }^{19,20}$. In addition, OC proteins have recently been shown to participate in the control of V2a IN diversification and migration, and to regulate in these neurons the expression of Pou $2 f 2^{13}$. However, other genes acting downstream of OC factors in the control of MN or IN development remain unknown.

To identify genes downstream of OC factors in MNs, we generated conditional Oc mutant embryos wherein $O c$ genes are specifically inactivated in MNs, and we compared the transcriptome of control and of conditional $O c$ mutant MNs using RNA sequencing (RNA-seq). Here, we show that lack of $O c$ expression in MNs leads to upregulation of $N k x 6.2$ expression in the LMC region. Surprisingly, by analyzing Oc constitutive mutants, we find that lack of OC factors in the spinal cord additionally downregulates $N k x 6.2$ expression in the V2a INs, indicating opposite effects of OC factors on Nkx6.2 in these two spinal neuron types. This suggests that Nkx6.2 acts downstream of OC factors in neuron populations that participate in the locomotor network, prompting us to test the requirement of Nkx6.2 for proper motor behavior including locomotion. Adult Nkx6.2-null mice exhibited some general motor deficits and specific locomotion defects characterized by altered coordination, abnormal stepping and fatigability, and $N k x 6.2$-null neonates exhibited shorter cycle periods and impaired alternation during non-weight-bearing locomotion (swimming). Analysis of fictive locomotion in Nkx6.2-null neonates demonstrated specific defects in CPG output. In the embryonic spinal cord of $N k x 6.2$-null mice, we observed a decrease in the number of LMCl MNs and alterations in the distribution of V2a INs. Adult Nkx6.2-null mice exhibited muscular hypertrophy in the portion of the quadriceps normally innervated by Nkx6.2 $2^{+}$MNs. Altogether, these results indicate that Nkx6.2 is regulated by OC factors in the embryonic spinal cord and is required for normal development of spinal locomotor and other motor circuits.

\section{Materials and methods}

Ethics statement and mouse lines. All experiments were performed strictly in accordance with the European Community Council directive of 24 November 1986 (86-609/ECC) and the decree of 20 October 1987 (87-848/EEC). Mice were raised in our animal facilities and treated according to the principles of laboratory animal care, and experiments and mouse housing were approved by the Animal Welfare Committee of Université catholique de Louvain (Permit Number: 2013/UCL/MD/11 and 2017/UCL/MD/008). The morning on which a vaginal plug was detected was defined as embryonic day (e) 0.5 . A minimum of three embryos ( $\mathrm{n} \geq 3$ ) of the same genotype was analyzed in each experiment. The embryos were harvested at embryonic days (e) 10.5, e12.5 or e14.5 depending on the mouse line, and staging was confirmed using conventional staging criteria. The $O c 1^{\text {flox/flox}} ; O c 2$ flox/flox16,21 mutant mice were crossed with Rosa26R-YFP/Olig2-Cre ${ }^{22}$ transgenic mice bearing heterozygous-null mutations for $\mathrm{Oc1}$ and $\mathrm{Oc} 2$ genes (Rosa26-YFP;Olig2Cre; $\mathrm{Oc1}^{+1-} ; \mathrm{Oc2}{ }^{+-}$), to obtain conditional double knockout of $O c 1$ and $O c 2$ in MNs. The combined inactivation of $O c 1$ and $O c 2$ in MNs completely abolished the expression of $O c 3^{10,11,15}$. Nkx6.2 $2^{+/ L a c Z}$ mouse embryonic stem cells ${ }^{23}$ were used to generate $N k x 6.2$ mutant mice $\left(N k x 6.2^{-/-}\right)$. To promote $L a c Z$ expression, $N k x 6.2$ mice were crossed with PGK-Cre mice to remove the neomycin resistance cassette flanked by LoxP sites located downstream of the LacZ sequence.

FACS, RNA purification, and RNA-sequencing. Spinal cords from e 10.5 control or cdKO mice were harvested and dissociated using a neural tissue dissociation kit (MACS; Miltenyi Biotec \#130-092-628) according to the manufacturer instructions. Dissociated cells were sorted by FACS (BD FACSAria III) to collect YFP-positive cells. Sorted cells were collected in TRIzol reagent, and RNA was purified with the Rneasy micro kit (QIAGEN \#74004). RNA concentration and quality were assessed using a Bioanalyzer (Agilent) and submitted to Genewiz to prepare an ultra-low input RNA-seq library before sequencing with an Illumina HiSeq. Preliminary data were analyzed by Genewiz using the standard RNA-seq data analysis package. RNAseq data have been deposited in the GEO repository (accession number: GSE141949).

In situ hybridization (ISH) and immunofluorescence. For ISH, collected embryos were immersion-fixed in ice-cold 4\% paraformaldehyde (PFA) in phosphate buffered-saline (PBS) overnight at $4{ }^{\circ} \mathrm{C}$, washed thrice in PBS for 10 minutes, incubated in PBS/30\% sucrose solution overnight at $4{ }^{\circ} \mathrm{C}$, and embedded and frozen in PBS/15\% sucrose/7.5\% gelatin. Fourteen- $\mu \mathrm{m}$ sections were prepared, and ISH was performed as previously described with DIG-conjugated Nkx6.2 antisense RNA probes (primer pair: $5^{\prime}$ GCTAAAAAGAAGCAAGACTCGG 3' and 5' CTCCGACGAGGACGTGTTAAA 3').

For immunofluorescence, collected embryos were immersion-fixed in 4\% PFA/PBS for 15, 25 or $35 \mathrm{~min}$ utes at $4{ }^{\circ} \mathrm{C}$ according to their embryonic stage, and processed as for ISH. Immunolabeling was performed on fourteen- $\mu \mathrm{m}$ serial cryosections as previously described ${ }^{18}$. Primary antibodies against the following proteins 
were used: BetaGal (chicken; 1:2000; Abcam \#ab9361), Chx10 (sheep; 1:500; Exalpha Biologicals \#X1179P), Er81 (rabbit; 1:10000; Covance), Foxp1 (goat; 1:1000; R\&D Systems \#AF4534), Gata3 (rat; 1:50; Absea Biotechnology \#111214D02), GFP (chick; 1:1000; Aves Lab \#GFP-1020), OC-1 (guinea pig; 1:2000 ${ }^{15}$; or rabbit; 1:100; Santa Cruz \#sc-13050; or sheep; 1:1000 R\&D Systems \#AF6277), Isl1 (goat; 1:3000; Neuromics \#GT15051), Lhx3 (mouse; 1:1000, DSHB \#67.4E12), MafA (guinea pig; 1:500; kindly provided by T. Müller), cMaf (rabbit; 1:5000; kindly provided by H. Wende), Nkx6.1 (mouse; 1:2000; DSHB \#F55A10), nNOS (rabbit, 1:4000; Immunostar \#24287), OC-2 (rat; 1:40024; or sheep; 1:500; R\&D Systems \#AF6294), OC-3 (guinea pig; 1:6000), Olig2 (rabbit; 1:2000; Millipore \#AB9610), RALDH2 (rabbit; 1:10000) and Shox2 (mouse; 1:500; Abcam \#AB55740). The secondary antibodies donkey anti-chicken/AlexaFluor 488, anti-guinea pig/AlexaFluor 594 or 647, anti-mouse/AlexaFluor 594 or 647, anti-rabbit/AlexaFluor 488, 594 or 647, anti-rat/AlexaFluor 488 or 647, anti-sheep/AlexaFluor 594 or 647 and goat anti-mouse IgG1 specific/AlexaFluor 594, purchased from ThermoFisher Scientific or Jackson Laboratories, were used at 1:2000 or 1:1000 dilution, respectively.

Immunofluorescence and ISH images from cryosections were acquired on an EVOS FL Auto Imaging System (ThermoFisher Scientific) or a confocal laser Scanning biological microscope FV1000 Fluoview using FV10-ASW 01.02 software (Olympus). The images were processed using Adobe Photoshop CS5 software to match brightness and contrast with the observations. For each embryo $(n \geq 3)$, neuron counts from one side of a minimum of five spinal cord sections at brachial, thoracic or lumbar levels were obtained using the count analysis tool of Adobe Photoshop CS5 software. Raw data were exported from Adobe Photoshop CS5 software to Sigma Plotv12.3 software to perform statistical analyses, and histograms were generated using Microsoft Excel. Appropriate statistical tests were applied depending on the number of comparisons and the data distribution and variance in each experimental group. For analysis of cell counts based on comparisons of two groups (control versus mutant), standard Student's or Welch's t-tests or Mann-Whitney U-tests were performed. Quantitative differences were considered significant at $\mathrm{p}<0.05$. Quantitative analyses of V2a IN spatial distributions were performed as previously described ${ }^{12}$. Briefly, in a transverse section of the spinal cord, height $(H)$ was defined as the distance from the ventral limit of the central canal to the dorsal-most edge of the spinal cord, and width $(W)$ as the distance from the central canal to the most lateral edge. For each V2a IN, distance $(\mathrm{dIN})$ and angle $(\alpha \mathrm{IN})$ were measured from the ventral limit of the central canal to the IN soma using the ruler analysis tool in Adobe Photoshop CS5 software. Relative dorso-ventral (DV) and medio-lateral (ML) positions of V2a INs were expressed as percentages of spinal cord height and hemicord width respectively: DV position and ML position were defined as (dIN $* \sin$ $\alpha \mathrm{IN}) / H$ and $(\mathrm{dIN} * \cos \alpha \mathrm{IN}) / W$, respectively (adapted from ${ }^{25}$ ), and ML versus DV values were plotted using Matlab software R2013a (Mathworks, Canada). Statistical analyses of ventral IN distribution were performed using a two-sample Hotelling's T2 test, which is a two-dimensional generalization of the Student's t-test. The analysis was implemented using the NCSS software package.

Histological analyses of hindlimb muscles. Quadriceps and gastrocnemius muscles were dissected at 2.5 or 5 months of age and weighed. They were immersion-fixed in 4\% PFA for 72 hours, paraffin-embedded and sectioned transversely at 20 micrometers. Sections were deparaffinized in Toluene $100 \%$ for $3 \times 5$ minutes, immersed in isopropanol $100 \%$ for 30 seconds and then rehydrated in a reverse ethanol series (100 to $30 \%, 2 \mathrm{~min}$ for each step). Sections were histologically stained with hemalun/eosin or labeled with Wheat Germ Agglutinine (WGA)/rhodamine (rabbit; 1:150; Vector RL-1022; Laboconsult) diluted in PBS and applied for 2 hours at RT. After washes in PBS, the slides were mounted as described above. Images were acquired with a Slide Scanner (3DHistech Pannoramic P250 Flash III) using CaseViewer software (3DHistech Ltd.) software. Central nuclei were counted on the entire RF portion of the quadriceps on at least 3 sections for each genotype $(n=3)$. For each muscle $(n=3)$, muscle fiber area was measured for at least 50 fibers in 5 sections using ImageJ software Raw data were exported to Microsoft Excel to draw the histograms.

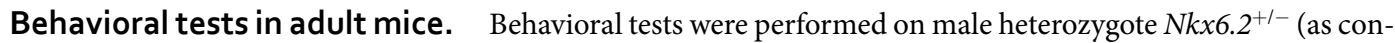
trol) or Nkx6.2-null mutant mice, starting at two months of age. All mice were handled and trained for at least two weeks before starting experiments, and weighed each experimental day. The rotarod test assesses the ability of mice to avoid falling from a rotating rod, and thus tests a conglomerate of motor skills including grip, balance and coordination. The Runway test evaluates the ability of mice to locomote across an elevated narrow runway towards their home cage. These two tests were performed as previously described ${ }^{14}$. The Catwalk test assesses gait during locomotion and was performed as previously described ${ }^{17}$. The wire test assesses grip fatigability and was performed as previously described ${ }^{26}$.

Behavioral tests in neonatal mice. The vestibulospinal reflex test specifically assesses the vestibulospinal reflex and was performed as previously described ${ }^{27}$ on $\mathrm{P} 4$ control or Nkx6.2-null mice. For each mouse, the test was performed ten times while video recording at 100 frames/sec. For the analysis, we wrote a script in ImageJ to compare the position of the hindlimb at the start of the rotation and at the end of the reflexive extension (see Supplemental Fig. 1). If no extension occured, the end-point measurement was made at $200 \mathrm{~ms}$. The script extracts the starting image (just before the rotation starts) and the image that the observer defines as the maximal hindlimb extension (or the image at $200 \mathrm{~ms}$ if no extension). The last image is then rotated $90^{\circ}$ counterclockwise to be in the same orientation as the starting image. A transparency overlay is created and the last image is manually re-aligned to the first. Lastly, the observer manually measures the distance between the paws of the overlaid images. The swimming test permits characterization of locomotor movements under non-weight-bearing conditions. The test was performed on P4 control or Nkx6.2-null mice. Briefly, the mouse pups were first habituated to the swimming chamber, which consisted of a $28 \mathrm{~cm}$ diameter glass dish containing $22^{\circ} \mathrm{C}$ water. On the test day, each pup was placed in the chamber and free swimming was recorded for $20 \mathrm{sec}$ with a NX3-S1 video camera (IDT, Tallahassee, FL, USA) fitted with a Canon EF-S $18-200 \mathrm{~mm}$ f/3.5-5.6 IS), using manufacturer-supplied 
software (Motion Studio) at $100 \mathrm{~Hz}$. The pup was then removed from the chamber, dried, warmed and returned to the litter. For analysis, a $10 \mathrm{sec}$ period containing the best swimming performance (typically in the middle of the 20 -sec session) was isolated and hindlimb tracking was performed using ImageJ- For each hindlimb, coordinates and timepoints were recorded at the moment of full flexion and full extension. Both the vestibulospinal reflex test and the free swimming test were done blinded to mouse genotype. All data were analyzed using ImageJ, and genotypes were disclosed at the end of the analysis.

Fictive locomotion analysis in neonatal mice. Neonatal mice (P3-P4) were deeply anesthetized with an overdose of Isoflurane, decapitated, and the entire spinal cord isolated in ice-cold oxygenated low $\mathrm{Ca}^{2+}$ artificial cerebrospinal fluid (ACSF, in mM: $128 \mathrm{NaCl}, 4 \mathrm{KCl}, 1 \mathrm{CaCl}_{2}, 1 \mathrm{MgSO}_{4}, 0.5 \mathrm{NaH}_{2} \mathrm{PO}_{4}, 21 \mathrm{NaHCO}_{3}, 30 \mathrm{D}$-glucose) as previously described ${ }^{28,29}$. The preparation was transferred to a recording chamber and continuously superfused thereafter with oxygenated normal-Ca ${ }^{2+}$ ASCF (in mM: $2 \mathrm{CaCl}_{2}$ ) at a rate of $2-5 \mathrm{ml} / \mathrm{min}$ at ambient temperature $\left(23-25^{\circ} \mathrm{C}\right)$. The preparation was left to rest for $30 \mathrm{~min}$ before recording. The electrophysiologist was blinded to mouse genotype until data analyses were completed.

Ventral root (L2 and L5) potentials were recorded using DPA differential amplifiers (NPI electronics, Germany), in AC-mode with bandpass filtering at $100 \mathrm{~Hz}-1.7 \mathrm{kHz}$, digitized at $10 \mathrm{kHz}$ (PicoScope $5442 \mathrm{~A}$, Pico Technology) and registered with a Picoscope 6 (Pico Technology, Cambrigeshire, UK) as previously described ${ }^{28,29}$. L2 and L5 ventral root discharges correspond predominantly to activity in hindlimb flexor and extensor MNs, respectively ${ }^{30,31}$. Alternating discharge in left versus right and L2 versus L5 ipsilateral ventral roots was interpreted to indicate locomotor CPG activity. Fictive locomotion (FL) was triggered by bath application of neurotransmitters /agonists, $\mathrm{N}$-methyl-D-aspartic acid (NMDA, $5 \mu \mathrm{M}$ ), serotonin (5-hydroxytryptamine creatinine sulfate, or 5 -HT, $10 \mu \mathrm{M})$, and dopamine (DA, $50 \mu \mathrm{M})$, as previously described ${ }^{28,29,32,33}$. Stock solutions of NMDA, serotonin, and dopamine were prepared fresh daily. At least $30 \mathrm{~min}$ of recording was performed for each preparation after drug application, with the last $10-15$ min of recording used for the analysis of FL after rhythm stabilization. FL was assessed in Nkx6.2-/ - and age-matched control preparations.

To analyze FL discharge patterns, a custom algorithm of quasi-periodic oscillation analysis was used as previously described ${ }^{28,29}$. Traces were filtered, rectified, resampled and smoothed using a weighted central moving average method (Hamming function) in Sigview (SignalLab) and SciDAVis (open-source and cross-platform, http://scidavis.sourceforge.net/). This represented bursting discharges as quasi-periodic wave oscillation events. The primary analysis of these oscillation events was performed using Clampfit 10.2 software (Axon Instruments) and parameters such as burst duration and cycle period were determined. Rhythm and inter-root phase relationships were further analyzed using two different methods: (1) circular analysis ${ }^{30}$, to obtain a statistical measure of L2-L5 coordination, and (2) quantification of phase relationships between flexor and extensor activity by cross-correlation analysis. The rhythmicity of bursts was assessed by autocorrelation analysis as previously described $^{29,31}$. Finally, the discharge frequencies of each individual root were analyzed. Five-fifteen min time intervals were used to plot frequency distribution graphs (normalized to peak value, bean distribution fitted by Kernel smooth fit).

Statistical testing. Statistical tests were applied based on the number of comparisons and the data distribution and variance in each group. Unless indicated elsewhere, for analysis based on comparisons of two groups (control or mutant), standard Student's t-tests or Mann-Whitney U tests were performed. Differences were considered significant at $\mathrm{p}<0.05$. To compare frequency distributions, the Kolmogorov-Smirnov two sample non-parametric test for empirical distributions was used.

\section{Results}

Generation of MN-specific Oc-deficient embryos. To identify genes downstream of OC factors possibly involved in $\mathrm{MN}$ diversification, we generated conditional mutant mice wherein $O c$ genes are specifically inactivated and the YFP fluorescent reporter is simultaneously expressed in MNs (Fig. 1A) (Olig2-Cre/Rosa26$Y F P / O c 1^{\Delta /-} O c 2^{\Delta /-}$ mice, referred to hereafter as cdKO mice). As previously described ${ }^{10-12,15}$, the deletion of $O c 1$ and $O c 2$ abrogated the expression of $O c 3$, enabling us to study the consequences of the absence of all 3 OC factors from MNs. We first evaluated the efficacy and specificity of the Cre recombinase to inactivate Oc genes in MNs. At e10.5, a majority of YFP-positive cells contained Isl1, a marker of spinal MNs. However, YFP was also detected in a neuron population ventral to MNs, corresponding to V3 INs (Fig. 1B), likely stemming from transient expression of Olig 2 in $\mathrm{p} 3$ progenitors during ventral spinal cord patterning ${ }^{34}$. In control embryos, OC factors were produced as soon as progenitors exited the cell cycle and their expression pattern overlapped in most of the newly born MNs (Fig. 1C,E) ${ }^{18}$. In cdKO mice, OC-1, OC-2 (Fig. 1D-D”) and OC-3 (Fig. 1F-F”) were lost in Isl1-positive MNs. Furthermore, Isl1 production was strongly decreased in the differentiating MNs located laterally, as previously demonstrated in constitutive Oc mutant mice (arrows in Fig. 1C', D') ${ }^{11}$. Thus, the Olig2-Cre allele efficiently eliminates the expression of $O c$ factors in MNs.

Nkx6.2 expression is differentially regulated by OC factors in MNs and INs. In an effort to identify genes downstream of OC factors during MN differentiation, we performed an RNA-sequencing comparison between control and cdKO YFP-positive cells at e10.5 (GEO repository accession number: GSE141949). Among genes showing a differential expression level (Supplemental Table 1) and compared to previous microarray experiments in e11.5 constitutive $O c$ mutant spinal $\operatorname{cord}^{13}$, the expression of $N k x 6.2$ was upregulated (1.32-fold increase) in cdKO YFP-positive cells as compared to controls, whereas it was downregulated (1.42-fold decrease) in the spinal cord of constitutive Oc mutant embryos ${ }^{13}$. Nkx6.2 (previously named Gtx) is a transcription factor involved in the ventral patterning of the embryonic spinal cord. In the p1 progenitor domain, Nkx6.2 represses 


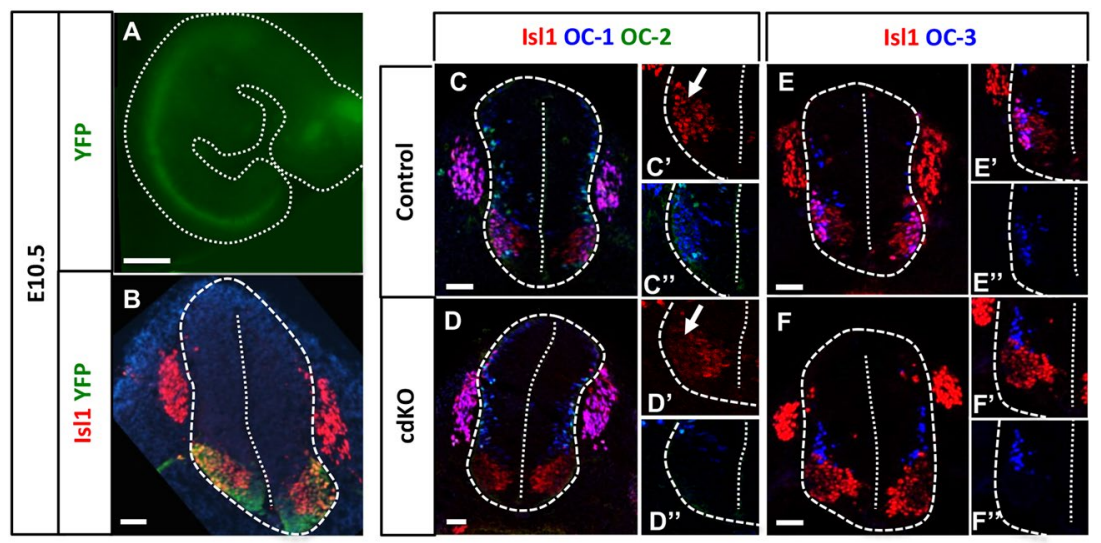

Figure 1. Validation of the conditional motor neuron-specific Oc-null mice. (A) YFP-positive cells in a whole Olig2-Cre/Rosa26-YFP/Oc1 ${ }^{\Delta /-} \mathrm{Oc} 2^{\Delta /-}$ embryo at e10.5, viewed from right side. The dotted line delineates the embryo, YFP fluorescence is observed along the spinal cord and in the encephalon. Scale bar $=1000 \mu \mathrm{m}$. (B) Immunostaining for YFP and Isl1 on a transverse section of control embryo at e10.5. Differentiating Isl1positive MNs contained YFP. YFP fluorescence was also detected in cells ventral to MNs, likely corresponding to V3 INs. (C-F) Immunostaining for Isl1, OC-1, OC-2 and OC-3 at e10.5 in control or conditional double-mutant $\left(O c 1^{-/} O c 2^{-/-}\right)$embryos. OC factor expression was lost in Isl1-positive MNs and Isl1 expression was decreased in the most laterally situated MNs (arrows). Scale bars $=20 \mu \mathrm{m}$.

$D b x 1$ expression and thereby allows the generation of $\mathrm{V} 1 \mathrm{INs}^{35-37} . N k x 6.2$ is also expressed in myelinating oligodendrocytes and in a specific motor pool of the lumbar $\mathrm{LMC}^{23,38}$.

To confirm the data from RNA-seq and microarray experiments and to understand the opposite regulation of $N k x 6.2$ in constitutive and conditional $O c$ mutants, we performed in situ hybridization for $N k x 6.2$ on transverse sections of control, constitutive (e11.5) or conditional (e12.5) Oc mutant spinal cords. In control embryos, Nkx6.2 transcripts were detected in the p1 domain (arrowheads in Fig. 2A-D, Fig. 2M-N) and ventral INs (black arrows in Fig. 2A-D, Fig. 2M-N) at brachial, thoracic and lumbar levels of the spinal cord, and in some MNs at brachial and lumbar levels (grey arrows in Fig. 2A-D, Fig. 2M,N). Previous analyses showed that the number of MNs in the LMC columns and of ventral INs in each cardinal population is unchanged in the absence of OC factors ${ }^{11,13}$. In constitutive $O c$ mutant $\left(O c 1 / O c 2^{-/-}\right)$embryos, $N k x 6.2$ expression was unchanged in the $\mathrm{p} 1$ domain but was lost in ventral INs (asterisks in Fig. 2E-H) and expanded to include more brachial and lumbar MNs (grey arrows in Fig. 2E,G,H). By contrast, in cdKO embryos, $N k x 6.2$ expression was unchanged in the p1 domain and in the ventral INs (arrowheads and black arrows in Fig. 2I-L), but expanded in brachial and lumbar MNs similar to the constitutive mutants (grey arrows in Fig. 2I,K,L). Thus, the consequences of the MN-specific inactivation of $O c$ genes on $N k x 6.2$ expression appeared to be specific to limb-innervating MNs, although the number of LMC neurons is not changed in the absence of OC factors ${ }^{11}$. To confirm that this expansion of $N k x 6.2$ expression was specific to LMC MNs, we combined at e12.5 in situ hybridization for Nkx6.2 (Fig. 2M-T) with immunofluorescence for the MN marker Isl1 (Fig. 2O,S) and for RALDH2, which is specifically expressed in LMC neurons ${ }^{39,40}$ (Fig. 2P,T). In mutant embryos, Nkx6.2 expression was coincident with, and masked, the RALDH2 immunolabeling (Fig. 2T), suggesting that Nkx6.2 was expressed within RALDH2-positive cells corresponding to LMC MNs. Taken together, these observations indicate that the dowregulation of $N k x 6.2$ expression in the constitutive mutant is due to elimination of expression in ventral INs despite expansion in LMC MNs ${ }^{11,13}$, whereas the upregulation seen in the cdKO mutant is due to expansion in LMC MNs without loss in ventral INs.

Absence of $N k \times 6.2$ results in general motor deficits and abnormal locomotion. Adult Nkx6.2-null mice exhibit motor coordination defects, which have been attributed to defective myelination in the $\mathrm{CNS}^{23}$, but more specific motor skills including locomotion were not investigated. To assess a possible requirement of Nkx6.2 expression for proper locomotion, we first reevaluated some general motor skills and then studied specific features of locomotion in adult $N k x 6.2$-null mice, compared to littermates that were heterozygous for the Nkx6.2 mutation (control mice). Control mice initially remained more than $35 \mathrm{~s}$ on the rotating rod, and this duration increased with repetition, indicative of learning. In contrast, $N k x 6.2$-null mice were not able to remain on the rotating rod more than $20 \mathrm{~s}$ on average, and no improvement was observed with time (Fig. 3A). Using the Runway test, control mice traversed the beam in $10 \mathrm{~s}$ on average with virtually no foot slips, and performance improved with repetition. In contrast, $N k \times 6.2$-null mice were slower and exhibited many foot slips, although both of these deficits improved substantially with repetition (Fig. 3B). Finally, the wire test revealed that $N k x 6.2$-null mice were more fatigable than control mice (Fig. $3 \mathrm{C} ; \mathrm{p}=0.014$ ). Thus, consistent with a previous report $^{23}, N k x 6.2$-null mice exhibited several general motor and motor learning deficits.

To assess whether specific locomotion-related abilities were altered, gait parameters were analyzed using the Catwalk assay. The duration to cross the catwalk was not different between control and Nkx6.2-null mice (Fig. 3D), indicating that the forward motion speed was normal. However, mutants used a larger number of steps $(6.4 \pm 0.22$ steps vs $5.6 \pm 0.16$ steps in control mice; $p=0.007)$ during the same period of time (Fig. $3 \mathrm{D})$, indicative 

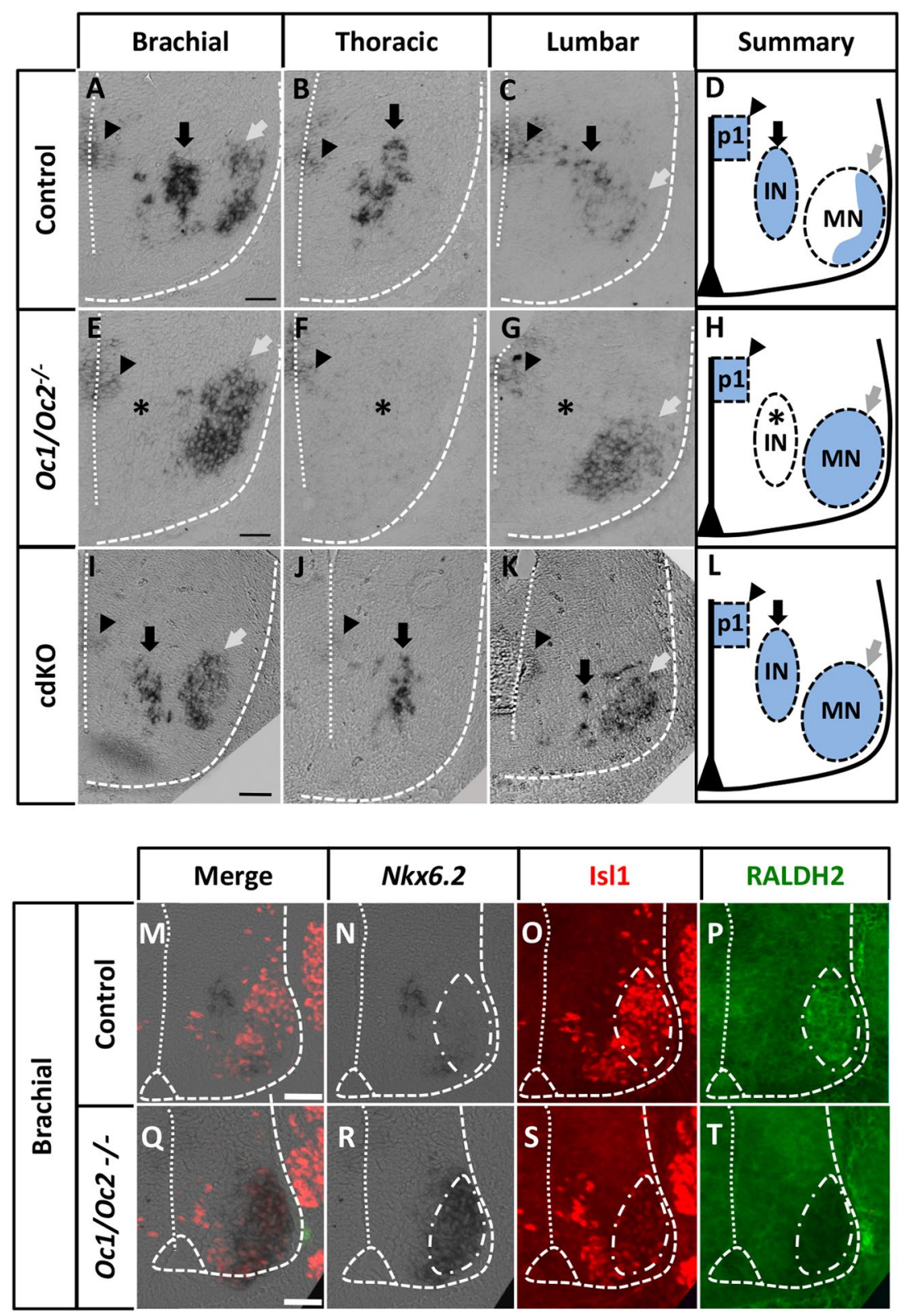

Figure 2. OC factors inversely regulate the expression of $N k x 6.2$ in motor neurons and in ventral interneurons. (A-I) In situ hybridization for Nkx6.2 on transverse sections at brachial, thoracic and lumbar levels of control or $O c$ constitutive double-mutant spinal cord at e11.5 or of conditional (cdKO) Oc mutant spinal cord at e12.5. $(\mathbf{D}, \mathbf{H}, \mathbf{L})$ shows a summary of $N k x 6.2$ expression (blue) in each genotype. (A-D) In control embryos, $N k x 6.2$ was expressed in p1 progenitors (arrowheads) and in ventral INs (black arrows) at all 3 levels, and in some MNs (grey arrows) at brachial and lumbar levels. (E-H) In the constitutive mutant, $N k x 6.2$ was present in the $\mathrm{pl}$ domain, lost in ventral INs (asterisks), but expanded in the brachial and lumbar MNs (grey arrows). (I-L) In the conditional mutant, $N k x 6.2$ was expressed in the p1 progenitors (arrowheads) and in ventral INs (black arrows), and expanded in brachial and especially lumbar MNs (grey arrows). (M-T) To determine if this expansion of Nkx6.2 expression was specific to MNs, we combined in situ hybridization for Nkx6.2 (Q-T) with immunofluorescence labeling for Isl1 (U-X) and RALDH2 (Y-BB) on transverse spinal cord sections at the brachial level of control or constitutive mutant at e12.5. RALDH2 labels all the limb-innervating LMC MNs. The Nkx6.2 in situ hybridization signal coincides with, and masks, RALDH2 immunostaining (which defines LMC MNs), indicating that $N k x 6.2$ expression in the Oc mutant embryos expanded to all the LMC cells. Scale bars $=100 \mu \mathrm{m}$.

of a higher gait frequency and a shorter stride length. Furthermore, Nkx6.2-null mice had fewer instances of simultaneous contacts with diagonal pairs of paws $(\mathrm{p}=0.04)$ and more instances of simultaneous contacts with three paws $(\mathrm{p}=0.008)$ (Fig. 3D), indicative of a change in gait pattern. 


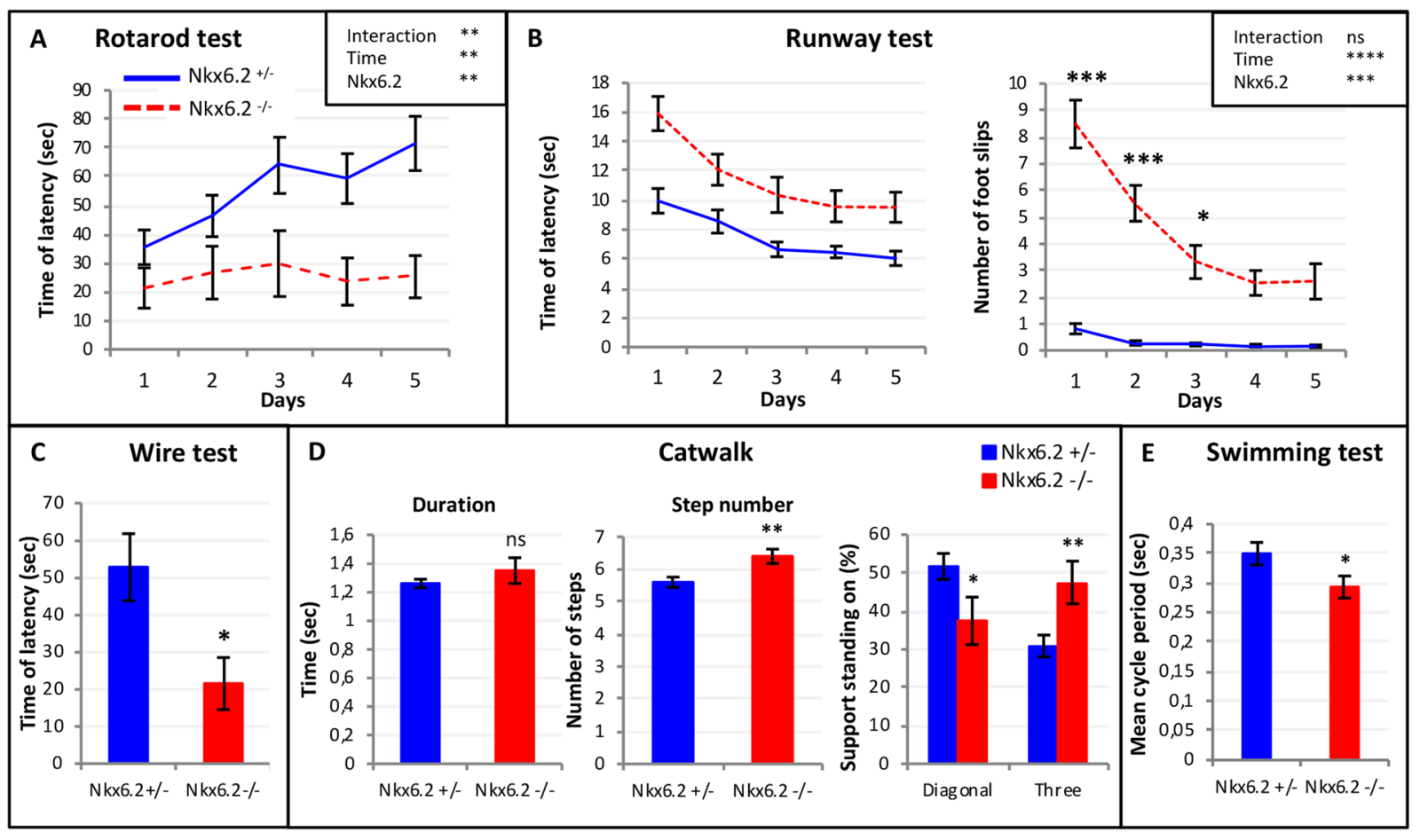

Figure 3. Motor deficits and abnormal locomotion in Nkx6.2-null mutant adult mice. (A) Behavioral tests were performed on $N k x 6.2$-null mice (Nkx6.2-I-) and compared to $N k x 6.2$ heterozygoous control mice $\left(N k x 6.2^{+/-}\right)$as controls. The rotarod test was performed on five successive days. $N k x 6.2$-null mice $(\mathrm{n}=14)$ were unable to stay on the rotating rod as long as heterozygous control mice $(n=18)$, and did not improve over time, as did the control mice. Genotype, time and interaction of both showed significant differences on the duration. (B) The Runway test was performed with heterozygous control $(\mathrm{n}=18)$ or $N k x 6.2$-null $(\mathrm{n}=14)$ mice for five successive days, and traversal time and number of foot slips were recorded. $N k x 6.2$-null mice traversed the runway more slowly and with more foot slips than control mice, although both groups improved over successive days. Genotype and time showed significant differences on the duration. (C) The wire test was used to test grip fatiguability. $N k x 6.2$-null mice were more fatigable than control mice (control: $\mathrm{n}=18$ vs mutant: $\mathrm{n}=14 ; \mathrm{p}=0.014)$. (D) Locomotion was tested on the catwalk. Locomotion in Nkx6.2-null mice $(\mathrm{n}=10)$ was characterized by an increased number of steps during the same period of time compared to control mice $(n=15)$, without any change in the speed of forward motion $(6.4 \pm 0.22$ steps vs $5.6 \pm 0.16$ steps in control mice; $\mathrm{p}=0.007)$. i.e. an increase in gait frequency and decrease in stride length. $N k x 6.2$-null mice also exhibited a decrease in the percentage of time with simultaneous contact with the substrate by diagonal paws $(\mathrm{p}=0.040)$ and an increase in the percentage of time of simultaneous contact by three paws $(\mathrm{p}=0.008)$, indicating a change in gait pattern. (E) To assess non-weight-bearing locomotion, the swimming test was performed on P4 Nkx6.2null mice $(\mathrm{n}=17)$ with heterozygote littermates as controls $(\mathrm{n}=11)$. Nkx6.2-null neonates had shorter mean cycle times $(\mathrm{p}<0.05)$.

To assess non-weight-bearing locomotion, and to bridge the behavioral studies of locomotion to the neonatal stages used for electrophysiological studies (see below), we assessed swimming in P4 Nkx6.2-null mice with heterozygote littermates as controls. $N k x 6.2$-null neonates had on average shorter mean cycle periods (Fig. 3E; $<$ <.05), in line with the increased gait frequency in adult Nkx6.2-null mice. We also noted that some Nkx6.2-null neonates, but no control mice, exhibited a locomotor pattern in which alternation was largely absent, with movements of fore- and hindlimbs on the same side dominating. This prompted us to examine the relationship between mean cycle period and deficient alternation more closely. We found that within the Nkx6.2-null group, the alternation-deficient locomotor pattern was exhibited by the Nkx6.2-null mice with the shortest mean cycle periods $(p=0.001)$. Moreover, comparison to the control group showed that the ratio of missed alternations to mean cycle period was significantly higher in the $N k x 6.2$-null group $(\mathrm{p}=0.037)$. Taken together, these data indicate that, in addition to general motor defects, $N k x 6.2$-null mice display altered locomotion characterized by increased step frequency and eroded locomotor pattern.

Nkx6.2-null neonates exhibit normal fictive locomotor patterning but altered cycle frequency distribution. Perturbed locomotor activity in adult and neonatal Nkx6.2-null mice notwithstanding, our behavioral observations do not pinpoint the locus of locomotor deficits. For example, they cannot formally exclude the possibility that the defective locomotor phenotype in Nkx6.2-null mice arises secondarily to myelination defects. To address this question, we assessed fictive locomotion (FL) on isolated Nkx6.2-null and heterozygous littermate control spinal cords, in which the locomotor CPG can be assessed directly, and at P3-P4, when 


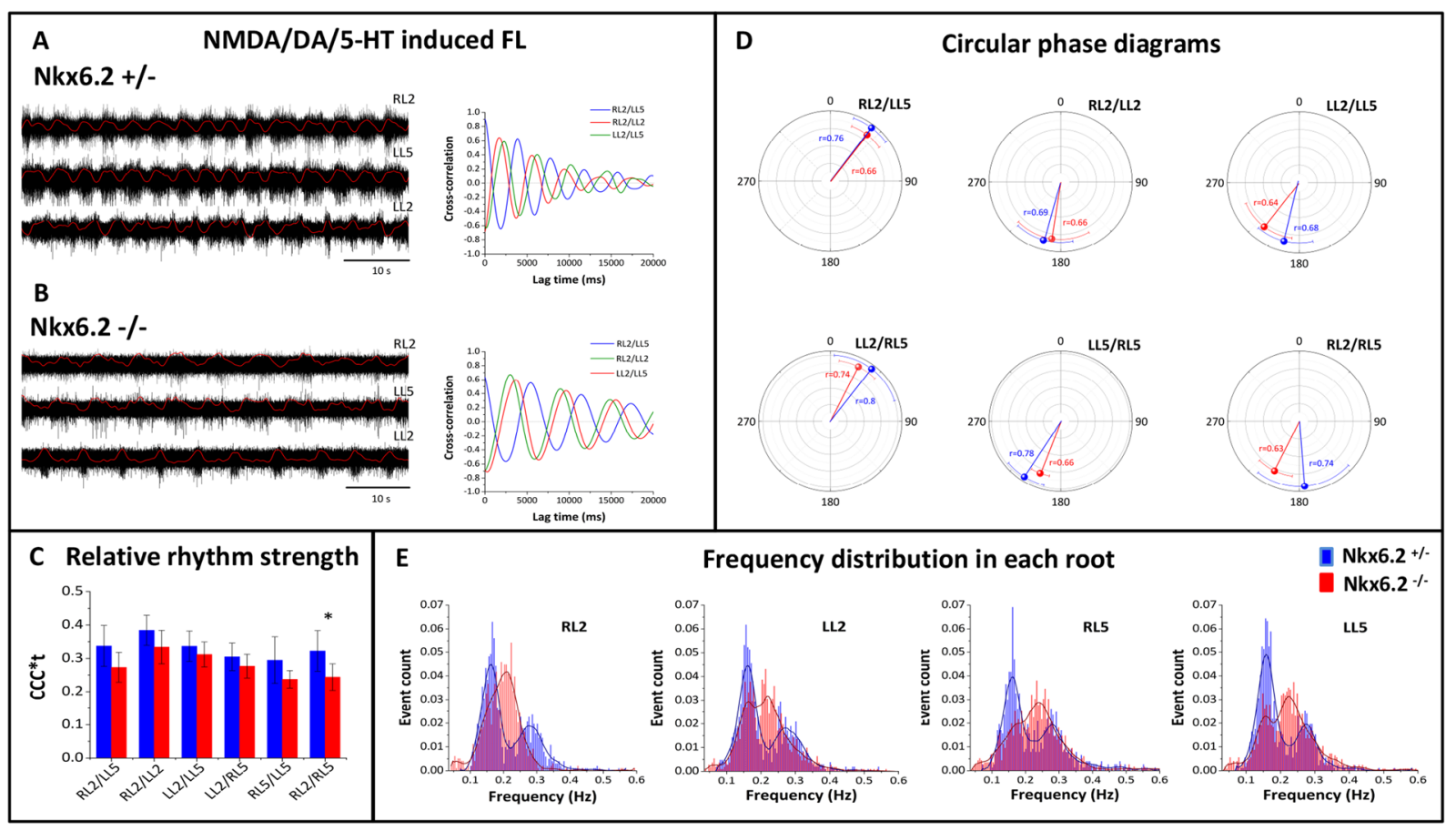

Figure 4. Fictive locomotion in control or $N k x 6.2$ mutant neonates. (A,B) Raw traces of fictive locomotion pattern in ventral roots (RL2, LL5, LL2) recorded in $N k x 6.2^{+/-}$(heterozygous control, A) or $N k x 6.2$-null (B) spinal cord preparations (right panel). Red trace represents rectified and smoothened version of the original trace (black). Cross-correlograms for corresponding root pairs are shown in the right panels. Time bar represents $10 \mathrm{~s}$. (C) Cross-correlation coefficient integral on time for all root pairs analyzed in $N k x 6.2^{+/-}$ (heterozygous control) or Nkx6.2-null preparations. (D) Circular phase diagrams show coordination between root pairs in $N k x 6.2^{+/-}$(heterozygous control, blue circles and error bars) and Nkx6.2-null (red circles and error bars) preparations. (E). Distributions of burst frequencies during fictive locomotion in each root (RL2, LL2, RL5, LL5) in Nkx6.2 $2^{+-}$(blue) and Nkx6.2-null (red) preparations.

central myelination is at an early stage ${ }^{23,41}$. There was no difference between control and $N k x 6.2$-null newborn mice in the vestibulospinal reflex test at P3-P4 (Supplemental Fig. 1), indicating no effect of the mutation on this descending pathway and suggesting no general central conduction velocity differences between mutants and controls at this neonatal stage.

FL was elicited by bath application of 5-HT/NMDA/DA $(10 \mu \mathrm{M}, 5 \mu \mathrm{M}$ and $50 \mu \mathrm{M}$ respectively), and recorded via the right (R) and left (L) L2 or L5 ventral roots. The typical pattern of FL with left/right and flexor/extensor alternation (RL2/LL2, LL2/LL5) and synchronous discharges for RL2/LL5 ventral roots was recorded in preparations of both genotypes (control $\mathrm{n}=8, N k x 6.2$-null $\mathrm{n}=11$; example of original traces for Nkx6.2 $2^{+/-}$and Nkx6.2-null shown in Fig. 4A,B). Cross-correlation analysis showed proper phase relationships in Nkx6.2-null preparations (Fig. 4B). Additionally, the cross-correlation coefficient (CCC) integrated over time was calculated to estimate rhythm strength for each root pair. As shown in Fig. 4C, there was no statistical difference in CCC analyzed for 3 of the pairs of roots between control and Nkx6.2-null preparations, the exception being the RL2/ RL5 root pair ( $\mathrm{p}=0.05$, Mann Whitney U-test).

Next, we examined the average phase relationships for different root pairs. As shown in Fig. 4D, there were no statistically significant differences between control and $N k x 6.2$-null preparations in the preferred phases for root pairs expected to be synchronous or alternating. For the synchronous pairs RL2/LL5 and LL2/RL5, mean preferred phases were respectively $37.2 \pm 17.3^{\circ}$ (control, $\mathrm{n}=5$ ) versus $37.9 \pm 17.0^{\circ}(\mathrm{Nkx6.2-null,} \mathrm{n}=7$ ) and $37.8 \pm 17.2^{\circ}$ (control, $\mathrm{n}=4$ ) versus $26.9 \pm 18.3^{\circ}(\mathrm{Nkx6.2}$-null, $\mathrm{n}=7$ ). For the alternating pairs RL2/LL2 and RL5/ LL5, mean preferred phases were respectively $196.1 \pm 27.5^{\circ}$ (control, $\mathrm{n}=7$ ) versus $188.7 \pm 37.3^{\circ}$ (Nkx6.2-null, $\mathrm{n}=7$ ) and $212.6 .1 \pm 18.2^{\circ}$ (control, $\mathrm{n}=4$ ) versus $201.2 \pm 9.8^{\circ}$ (for $N k x 6.2$-null, $\mathrm{n}=8$ ). For the ipsilateral alternating pairs LL2/LL5 and RL2/RL5, mean preferred phases were respectively $194.2 \pm 27.0^{\circ}$ (control, $\mathrm{n}=6$ ) versus $217.6 \pm 30.3^{\circ}\left(\mathrm{Nkx6.2}\right.$-null, $\mathrm{n}=7$ ) and $175.3 \pm 43.0^{\circ}$ (control, $\mathrm{n}=5$ ) versus $206.0 \pm 19.0^{\circ}(\mathrm{Nkx6.2}$-null, $\mathrm{n}=6$ ). Although left-right and flexor-extensor alternation was not affected in Nkx6.2-null preparations, extra burst activity was sometimes recorded in RL5 (less often in LL5) roots, which could lead to a phase drift for inter-segmental alternation in mutants (data not shown).

Finally, we analyzed the frequency distribution of bursts. As shown in Fig. 4E, in control preparations we observed two frequency peaks for each root, a slow frequency peak at about $0.16 \mathrm{~Hz}$ and a fast frequency peak at about $0.27 \mathrm{~Hz}$. In contrast, in $N k x 6.2$-null preparations, we observed only a single, intermediate frequency peak at about $0.22 \mathrm{~Hz}$, but in some cases with a lower frequency shoulder. The frequency distributions for the RL2 and LL2 roots differed significantly between $N k x 6.2+I-$ and $N k x 6.2$-null preparations (Kolmogorov-Smirnov test, 

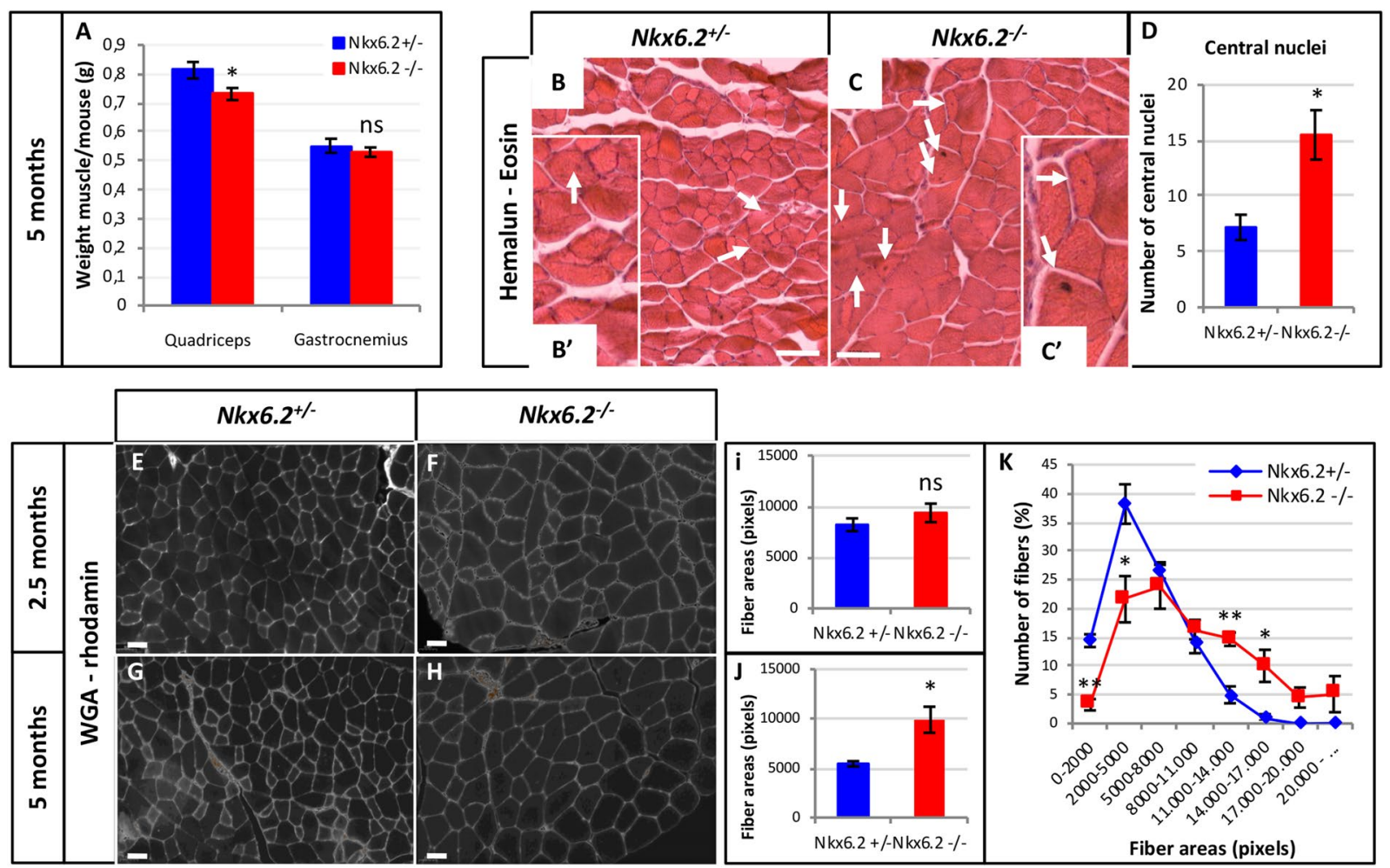

Figure 5. Absence of $N k x 6.2$ results in Rectus Femoris muscle alterations. (A) Quadriceps and gastrocnemius muscles from $N k x 6.2$ heterozygous $(+/-)$ or null $(-/-)$ mice at 5 months of age were weighted and normalized to the body weight. The weight of the quadriceps was lower in Nkx6.2-null mice than in heterozygous control mice $(n=7 ; p=0.038)$, while the weight of the gastrocnemius muscle was unchanged. (B-D) Hemalun/eosin labelings of transverse sections in the Rectus Femoris portion of the quadriceps from heterozygous control or Nkx6.2-null mice at five months of age. Insets show higher magnifications. The number of central nuclei (white arrows) was increased in the Rectus Femoris portion in the absence of $N k x 6.2(n=3 ; \mathrm{p}=0.016)$. Scale bar $=100 \mu \mathrm{m}$. (E-J) Wheat-Germ Agglutinin (WGA)/rhodamin labelings of transverse sections in the Rectus Femoris portion of the quadriceps muscle from heterozygous control or Nkx6.2-null mice at 2.5 or five months of age. Rectus Femoris fibers were larger in the absence of $N k x 6.2$ at five months of age. Scale bar $=50 \mu \mathrm{m}$. $(\mathbf{K})$ Distribution of fiber area in the Rectus Femoris portion of heterozygous control and mutant mice at 5 months of age. Nkx6.2-null mice exhibited a higher number of large diameter fibers compared to control mice $(\mathrm{n}=3)$.

$p<0.001)$. Significance was not reached for the RL5 and LL5 roots $(p=0.159$ and $p=0.160$ respectively). We also pooled the peak frequencies for all roots in the two groups $(n=3754$ for controls $n=4510$ for mutants), and found a similar differential distribution, with one pronounced peak for $N k x 6.2$-null preparations and a bi-modal distribution for control preparations (Kolmogorov-Smirnov test, $\mathrm{p}<0.001$; Fig. 4D).

Taken together, these observations demonstrate that $N k x 6.2$-null spinal cord preparations can generate normally patterned FL, but tend to have intermediate burst frequencies relative to heterozygous preparations. This might be related to the observation that heterozygous adult and neonatal mice have lower average gait frequencies and swim cycle frequencies than their Nkx6.2-null littermates.

Absence of $N k \times 6.2$ results in muscle fiber hypertrophy in a portion of the quadriceps. Altered formation or activity of spinal locomotor circuits often results in subsequent changes in innervation or morphology of the corresponding muscles. At hindlimb levels of the spinal cord, Nkx6.2 defines a specific motor pool supplying innervation to the Rectus Femoris (RF) portion of the quadriceps muscle (Fig. S2) ${ }^{38}$. Innervation of the RF was previously reported to be normal in Nkx6.2-null animals ${ }^{38}$, and we observed here that the density of neuro-muscular junctions was comparable in Nkx6.2-null mice and heterozygous control littermates (data not shown). In contrast, the weight of the quadriceps normalized to body weight was reduced in Nkx6.2-null mice compared to controls, whereas the weight of the gastrocnemius muscle was similar (Fig. $5 \mathrm{~A} ; \mathrm{n}=7 ; \mathrm{p}=0.038$ ). In addition, the number of central muscle fiber nuclei in the RF portion of the quadriceps was increased in Nkx6.2-null mice (arrows in Fig. $5 \mathrm{~B}-\mathrm{D} ; \mathrm{n}=3 ; \mathrm{p}=0.016$ ), suggesting that it had undergone repetitive degeneration/regeneration cycles ${ }^{42}$. Furthermore, the mean cross-sectional area of RF fibers showed no change at 2.5 months of age (Fig. 5E,F,I) but an increase in $N k x 6.2$-null mice by 5 months of age (Fig. 5G,H,J n $=3 ; \mathrm{p}=0.03$ ). Consistently, the distribution of fiber area at 5 months of age showed that RF portion of the quadriceps in Nkx6.2-null mice was composed of larger fibers than in control mice (Fig. $5 \mathrm{~K} ; \mathrm{n}=3$ ). Thus, motor deficits in Nkx6.2-null mice are accompanied by changes in a specific hindlimb muscle, suggesting a specific effect on the respective MNs. 


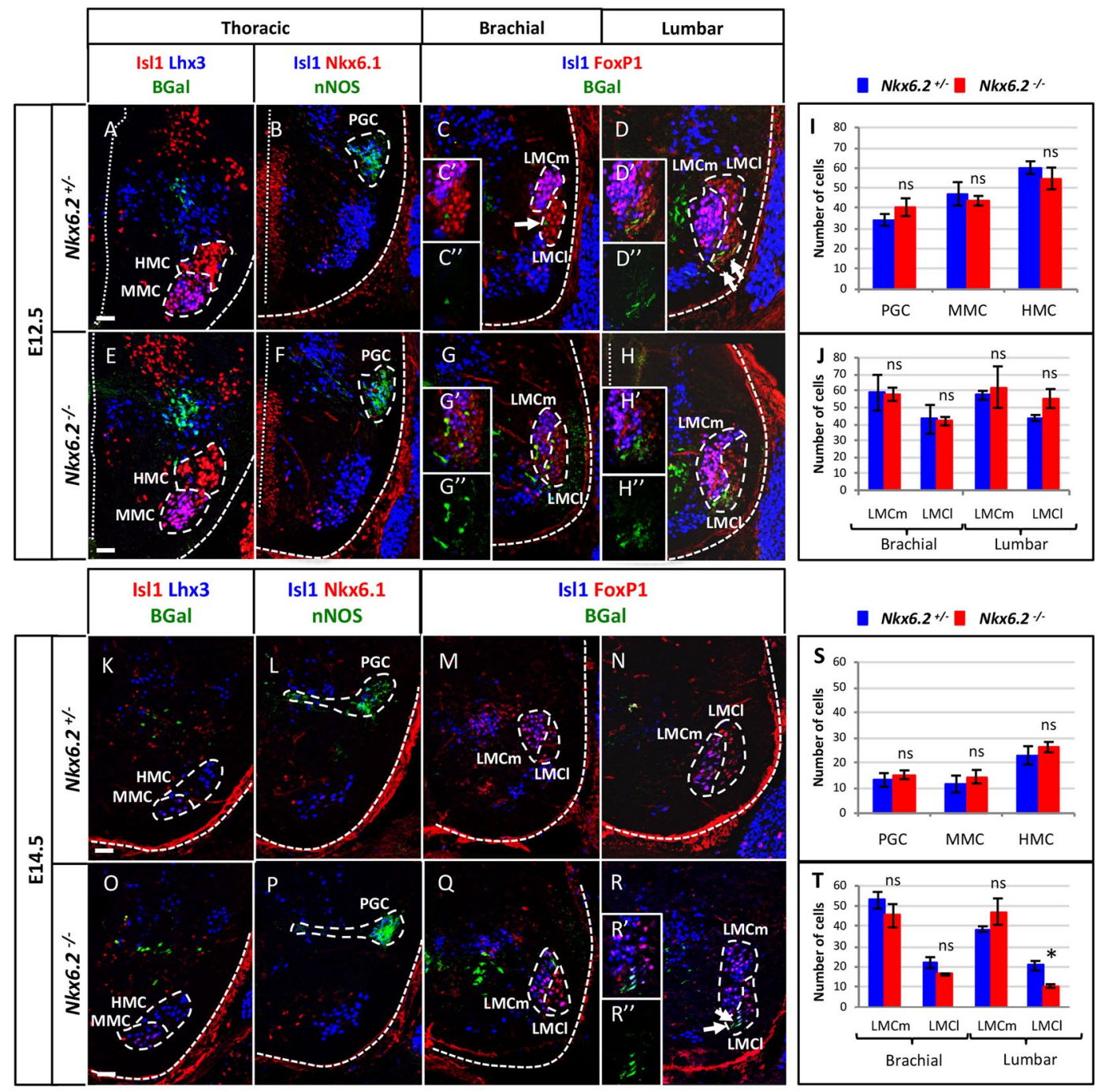

Figure 6. $N k x 6.2$ is required for the maintenance of $\mathrm{LMCl}$ motor neurons. Immunostaining of transverse spinal cord sections of $N k x 6.2$ heterozygous control or $N k x 6.2$-null mutant embryos at e12.5 (A-J) or e14.5 (K-T). (A-J) In the heterozygous control embryos at e12.5, $\beta$-galactosidase was absent from HMC (Isl1+), MMC (Isl1 + Lhx3 + ) and PGC (Isl1 + nNos + ) at thoracic level, but was detected in rare MNs of the LMCl (Foxp1 + Isl1-) at brachial level (arrow in C) and in a greater number of $\mathrm{LMCl}$ cells at lumbar level (arrows in D). In Nkx6.2-null mutants, the $\beta$-galactosidase signal was observed in more LMCl MNs at brachial (arrows in $\mathbf{G}$ ) and at lumbar (arrows in $\mathbf{H}$ ) levels. The production of HMC, MMC and PGC MNs was not affected in the absence of $N k x 6.2$. Similarly, no change was detected in the LMC at brachial or lumbar levels of the spinal cord. (K-T) At e14.5, $\beta$-galactosidase signal was only detected in some LMCl MNs at lumbar level (arrows in R) in Nkx6.2 mutant mice. The production of HMC, MMC and PGC MNs at thoracic level was not changed by absence of $N k x 6.2$. In contrast, the number of $\mathrm{LMCl} \mathrm{MNs}$ at lumbar level was significantly decreased in the Nkx6.2-null mice $(\mathbf{N}, \mathbf{R}, \mathbf{T})$ with no perturbation of the LMC at brachial level and of the LMCm (Foxp1 + Isl1 +) at lumbar level. $\mathrm{n} \geq 3 ; * \mathrm{p}<0.05$. Scale bar $=50 \mu \mathrm{m}$.

Nkx6.2 is necessary for proper maintenance of LMCI MNs at lumbar level. Although Nkx6.2 and its paralog factor Nkx6.1 are critical for ventral neural tube patterning, the absence of $N k x 6.2$ in spinal progenitors is compensated by the presence of Nkx6.1. Therefore, $N k x 6.2$ single mutants do not exhibit ventral patterning defects ${ }^{35,43}$, enabling us to study its role at later stages of spinal neuron development. Accordingly, we ascertained which neuron types depend on Nkx6.2 during later development, beginning with MNs. Previous studies have demonstrated Nkx6.2 expression in MNs of the $\mathrm{LMCl}$, which is characterized by expression of Foxp1 but not Isl $1^{38}$. In the thoracic spinal cord of heterozygous $N k x 6.2^{+/-}$embryos at e12.5, $\beta$-galactosidase was absent from the somatic HMC and MMC MNs (Fig. 6A) as well as from the nNOS-positive visceral MNs (Fig. 6B), as expected $^{38}$. At limb levels, although the $\beta$-galactosidase signal was weak, it was detected in a few MNs of the brachial LMCl (arrow in Fig. 6C), and a greater number of MNs in the most ventral part of the lumbar LMCl (arrows in Fig. 6D). In Nkx6.2-null embryos, a stronger $\beta$-galactosidase signal was detected in more of the brachial LMCl MNs. These $\beta$-galactosidase + MNs were interspersed with LMCm neurons (characterized by the expression of both Foxp1 and Isl1; arrows in Fig. 6G-G"), consistent with a previous report showing that MNs expressing Nkx6 
proteins are initially intermixed within the LMC before segregating into coherent clusters ${ }^{38}$. In the lumbar spinal cord of Nkx6.2-null mutants, $\beta$-galactosidase-positive MNs were located in the most ventral part of the LMCl (arrows in Fig. 6H-H") as in controls (Fig. 6D). At e14.5, the $\beta$-galactosidase signal was weaker and was only detected in some LMCl MNs in the lumbar Nkx6.2-null spinal cord (arrows in Fig. 6R). Thus, consistent with previous reports ${ }^{38}$, Nkx6.2 in differentiating MNs is restricted to a subset of LMCl MNs likely corresponding to a specific motor pool. The presence of $\beta$-galactosidase-positive MNs in the LMC of Nkx6.2-null spinal cords suggests that $N k x 6.2$ expression is not required for the generation of the Nkx6.2+MNs.

To evaluate if the absence of $N k x 6.2$ impacts MN subsets, motor columns were studied at e12.5 (Fig. 6A-J) and e14.5 (Fig. 6K-T). At e12.5, no change was observed at thoracic levels in the number and location of MNs in the HMC (Isl1+), MMC (Isl1 + Lhx3+) (Fig. 6A,E,I) or PGC (Isl1 + nNOS+) (Fig. 6B,F,I), or at limb levels in LMCm (FoxP1 + Isl1+) or LMCl (FoxP1 + Isl1-) MNs (Fig. 6C,D,G,H,J). At e14.5, the developmental stage during which motor pools are specified, no change was observed in HMC, MMC (Fig. 6K,O,S) or PGC (Fig. 6L,P,S) MNs at thoracic levels or in either division of the LMC at brachial levels (Fig. 6M,Q,T). In contrast, the number of LMCl MNs was significantly decreased at lumbar levels in the $N k x 6.2$-null spinal cords (Fig. $6 \mathrm{~N}, \mathrm{R}, \mathrm{T} ; \mathrm{n}=3$; $\mathrm{p}=0.017$ ). Taken together, these observations suggest that $N k x 6.2$ expression is not required for the production of Nkx6.2-expressing LMC MNs, but is required for the survival of a discrete subset of LMCl cells.

To determine if the decrease in the number of $\mathrm{LMCl}$ MNs at lumbar level at e14.5 correlated with the absence of specific motor pools, the distribution of Nkx6.1 and Er81 was studied at e14.5. Nkx6.1, the paralog of Nkx6.2, is expressed in different motor pools within the LMCm whereas Er81, an ETS family protein, is expressed in motor pools within both divisions of the $\mathrm{LMC}^{38,44}$. Pea3, another ETS protein, has been shown to be co-expressed with Nkx6.2 within the $\mathrm{LMCl}^{38}$, but due to a lack of an effective Pea3 antibody, we were unable to characterize this MN subpopulation. Nonetheless, the number of Nkx6.1 + nor Er81 + MNs was unaltered in the lumbar LMC of Nkx6.2-null embryos (Fig. S3), suggesting that the absence of Nkx6.2 in the LMCl does not impact on the generation or maintenance of Nkx6.1 or Er81 motor pools but results in the loss of another LMC neuron subset that remains to be identified.

Nkx6.2 regulates the distribution of $\mathrm{V} 2 \mathrm{a}$ INs during spinal cord development. The deficits in motor behavior and changes in FL observed in Nkx6.2-null mice suggest that some aspects of the coordination of MN activity are altered. Therefore, we investigated further the role of Nkx6.2 in ventral IN development. In addition to MNs and the $\mathrm{p} 1$ domain $^{35,37}, N k x 6.2$ expression was detected in the V2 IN domain in the ventral spinal cord (Fig. 2A-D). To determine whether $N k x 6.2$ is expressed in V2 INs, we compared the distribution of $\beta$-galactosidase with that of V2 markers in heterozygous $N k x 6.2^{+/-}$embryos at e12.5. Although the signal was weak, $\beta$-galactosidase was present in cells containing Chx10 corresponding to V2a INs (arrows in Fig. 7A). In contrast, $\beta$-galactosidase was never detected in Gata3 + V2b INs or in other IN populations (Fig. $7 \mathrm{~A}$ and data not shown). In Nkx6.2-null embryos, the $\beta$-galactosidase signal was stronger and was co-detected with Chx10 in V2a INs (arrows in Fig. 7B), confirming expression of $N k x 6.2$ in V2a postmitotic INs.

OC factors, which regulate the diversification and the distribution of $\mathrm{V} 2 \mathrm{a} \mathrm{INs}{ }^{13}$, are thus required for $\mathrm{Nkx6.2}$ expression in V2a INs (Fig. 2E-H). To assess a possible function for Nkx6.2 in V2a development, we characterized the phenotype of V2a INs in $N k x 6.2^{-I-}$ embryos at e12.5 $(\mathrm{n}=3)$. In the absence of $N k x 6.2$, the total number of Chx10 + V2a INs was moderately decreased (16\%) at the thoracic level, but was indistinguishable from controls at brachial or lumbar levels (Fig. 7C-E). Among the V2a subpopulations, none of the Shox $2+$, $\mathrm{MafA}+$ or $\mathrm{cMaf}+$ subsets were altered in the absence of $N k x 6.2$ (Fig. 7F-N). Hence, Nkx6.2 is not required for the diversification of the V2a INs but appears to be necessary for appropriate production of a subset of V2a INs at the thoracic level.

However, careful examination of the Chx10 immunostaining suggested that Nkx6.2 might contribute to the regulation of V2a IN position (Fig. 7C-D). Therefore, a quantitative comparison of the distribution of V2a INs was performed in control and in $N k x 6.2$-null mutants. In heterozygous $N k x 6.2^{+1-}$ embryos, V2a INs were distributed between two interconnected clusters, a major central group, and a minor medial group, at each level of the spinal cord (Fig. 8A-C). In mutant embryos, the relative cell distribution between these clusters appeared altered (Fig. 8D-L). At brachial levels, the two clusters were more segregated with more cells in the medial group (Fig. 8D,G-H; $\mathrm{n}=3 ; \mathrm{p}<0.001$ ). At thoracic levels, the number of V2a INs was increased in the central group (Fig. 8E,I-J; $\mathrm{n}=3 ; \mathrm{p}<0.001$ ). Finally, at lumbar levels, the two clusters were less segregated than in control embryos (Fig. 8F,K-L; $n=3 ; \mathrm{p}<0.05$ ). Taken together, these results demonstrate that Nkx6.2 is not required for the production of V2a INs but influences their ultimate distribution within the ventral part of the spinal cord.

\section{Discussion}

Several prior studies have identified a contribution of the OC factors to MN and IN development in the embryonic spinal cord ${ }^{11-13,16,19,20}$. Here, we show that OC factors differentially regulate the expression of $N k x 6.2$ in V2a INs and MNs. We provide evidence that Nkx6.2 is required for proper motor function and locomotor behavior, and we demonstrate that Nkx6.2 is necessary for the survival of a subset of LMC MNs and for the proper distribution of V2a INs. We have thus identified Nkx6.2 as a novel regulator of spinal motor circuit formation downstream of the OC factors (Fig. 9).

Our observations indicate that OC transcription factors stimulate the expression of $N k x 6.2$ in V2a INs while restricting it within LMC MNs. Consistently, results of a microarray experiment on constitutive Oc-null mutant spinal cord revealed that $N k x 6.2$ was decreased in the absence of OC factors ${ }^{13}$, while RNA-seq comparison from OC-deficient MNs showed an increase in Nkx6.2 expression. Three hypotheses could account for these observations. First, changes in the expression levels of $N k x 6.2$ in specific spinal populations of $O c$ mutant embryos may be secondary to alterations in the identity or survival of these cells. In the absence of $O c$ factors, identity of the $\mathrm{LMCm} \mathrm{MNs}$ is converted into that of the $\mathrm{LMCl}$ cells due to defective maintenance of Isl 1 expression, resulting in 

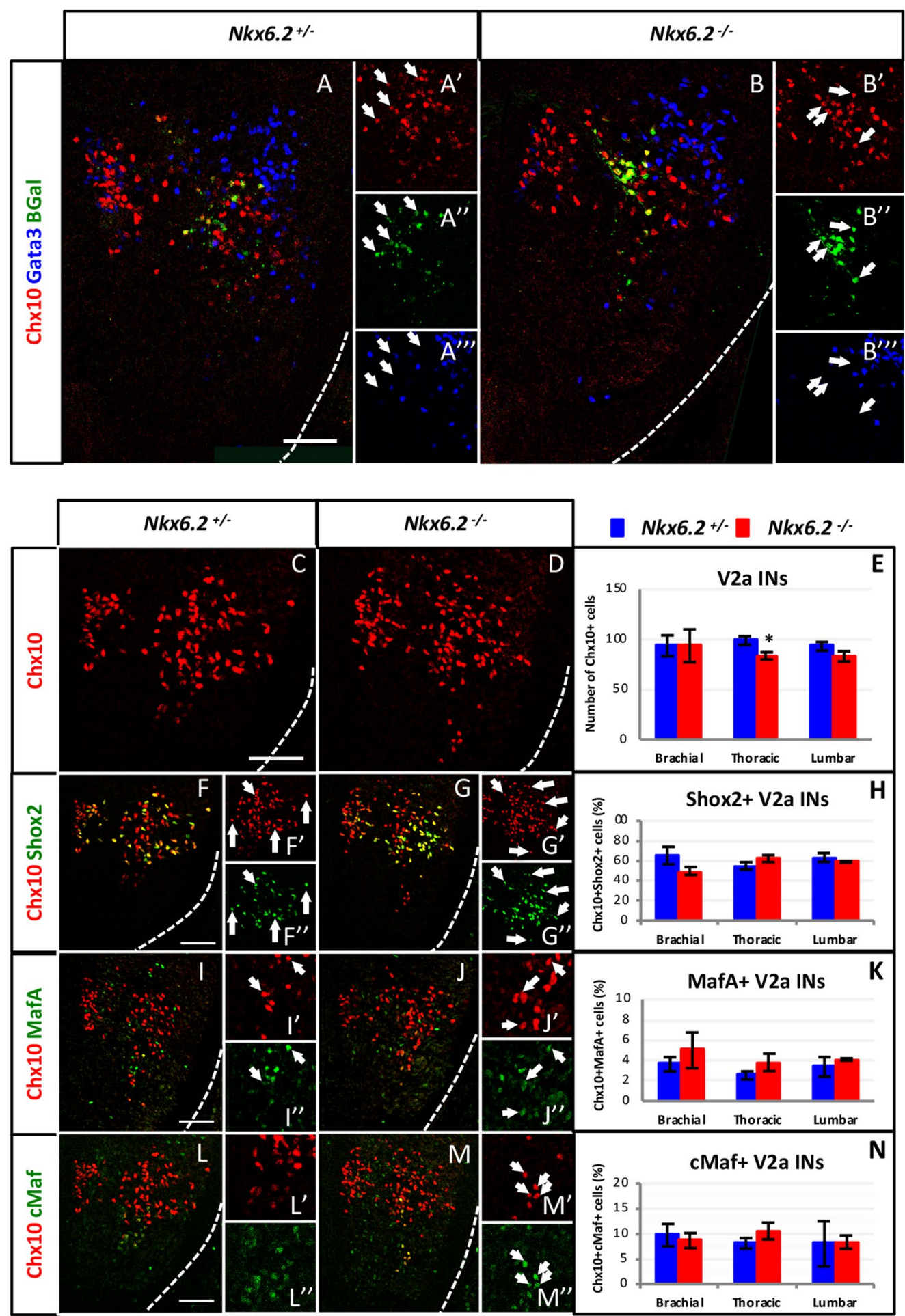

Figure 7. Nkx6.2 is not necessary for proper diversification of V2a interneurons. Immunostaining of transverse brachial spinal cord sections of $N k x 6.2$ heterozygous control or Nkx6.2-null mutant embryos at e12.5. (A) In heterozygous control embryos, $\beta$-galactosidase was present in Chx10-positive cells corresponding to V2a INs (arrows), but was absent from Gata3 + V2b INs. (B) Similar observations were made in the Nkx6.2-null mutant embryos (arrows). (C-E) In the absence of Nkx6.2, the number of V2a INs was modestly decreased at the thoracic level, but not at the brachial or lumbar levels. (F-N) Among the examined V2a IN subpopulations, generation of the Shox $2+(\mathbf{F}-\mathbf{H})$, MafA $+(\mathbf{I}-\mathbf{K})$ and $c M a f+(\mathbf{L}-\mathbf{N})$ subsets was unaffected. $\mathrm{n}=3 ; * \mathrm{p}<0.05$. Scale bars $=50 \mu \mathrm{m}$. 


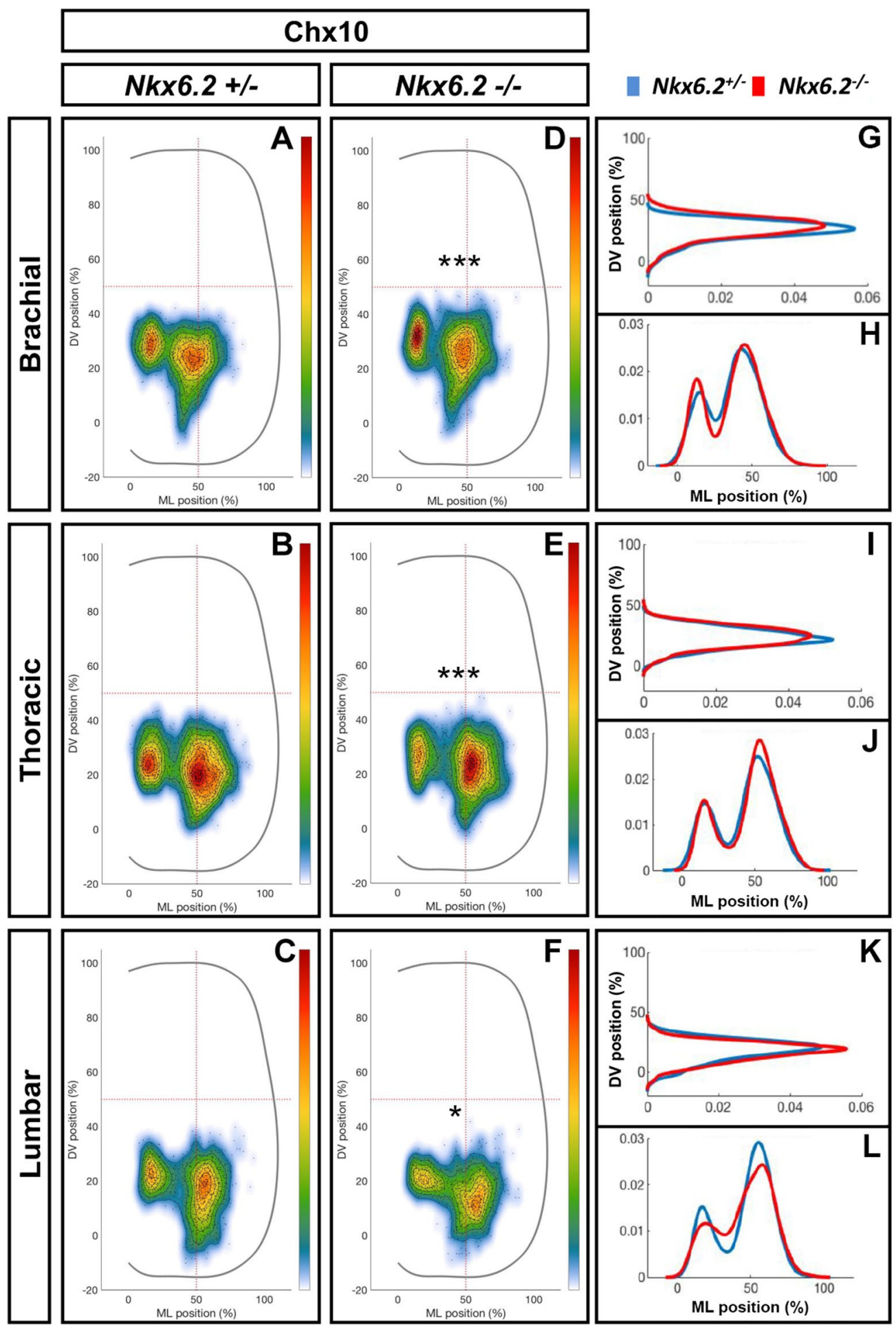

Figure 8. Nkx6.2 contributes to the regulation of the V2a interneuron distribution. Integrated distribution of V2a INs in the transverse plane of the spinal cord in Nkx6.2 heterozygous control or Nkx6.2-null mutant embryos at e12.5 (only the right half of the spinal cord is shown). (A-F) Two-dimensional cell density heatmaps show the integration of V2a IN distribution from multiple sections from multiple embryos of each genotype. The color code represents cell density. (G-L) One-dimensional graphs (right) compare density distribution (arbitrary cell density units) in control (blue) and in mutant embryos (red) along the dorso-ventral (DV) or the medio-lateral (ML) axes of the spinal cord. (A-C) In Nkx6.2 heterozygous control embryos, V2a INs are distributed in two connected clusters, a major central group and a minor medial group, at each level of the spinal cord. (D-L) In Nkx6.2-null embryos, the relative cell distribution between the two clusters is altered with greater separation of clusters and more cells in the medial group at the brachial level (D, G-H), more cells in the central group at the thoracic level (E, I-J), and fewer cells in less separated clusters at the lumbar level (F, K-L). $\mathrm{n}=3 ; \mathrm{p}<0.001$ at brachial and thoracic levels, $\mathrm{p}<0.05$ at lumbar level. 


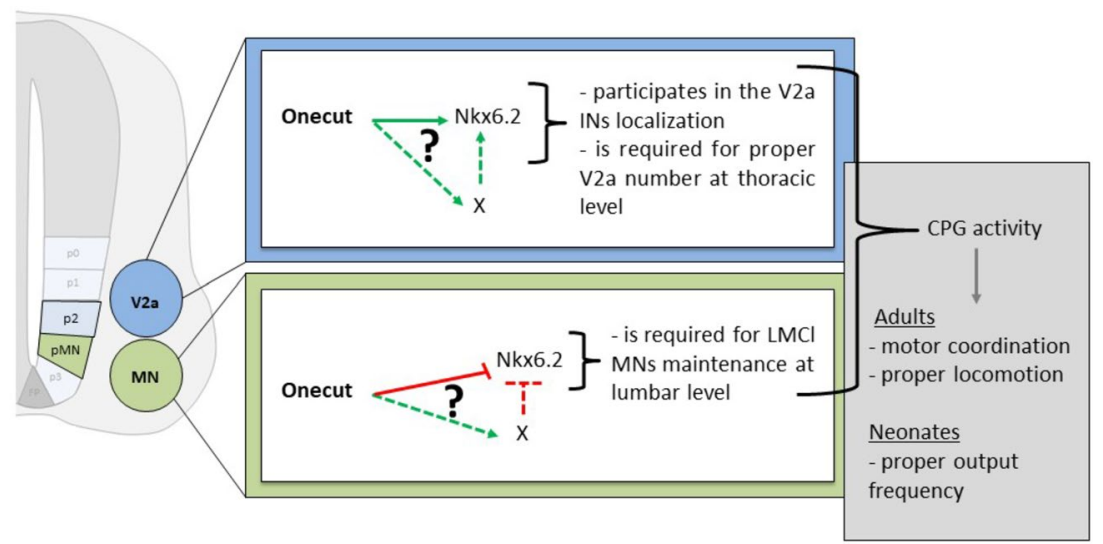

Figure 9. Working model for OC factor and Nkx6.2 contribution to CPG development. OC factors directly or indirectly stimulate $N k x 6.2$ expression in V2a INs wherein Nkx6.2 regulate cell number and distribution. In contrast, OC factors directly or indirectly repress Nkx6.2 expression in LMC MNs wherein Nkx6.2 is necessary to maintain the number of $\mathrm{LMCl}$ cells at lumbar level. The combined action of Nkx6.2 in V2a INs and MNs contribute to proper CPG formation and activity at both the neonatal and the adult age. Green: stimulation, red: inhibition, dotted lines: indirect regulations.

an expansion of $\mathrm{LMCl} \mathrm{MNs}^{11}$. However, $\mathrm{Nkx} 6.2$ is restricted to a ventral pool of $\mathrm{LMCl} \mathrm{MNs}$ in control embryos ${ }^{38}$ but is detected in all the LMC cells in Oc-deficient embryos. Conversely, Nkx6.2 expression was strongly downregulated in V2a INs of $\mathrm{Oc}$ mutant embryos whereas the total number of V2a was unaffected ${ }^{13}$. Therefore, it seems unlikely that perturbations of $N k x 6.2$ expression in the absence of $\mathrm{OC}$ factors may be secondary to differentiation or survival defects. Second, OC factors may regulate $N k x 6.2$ in MNs and INs by different mechanisms. OC factors are described as transcriptional activators ${ }^{45-48}$. Thus, they could directly stimulate $N k x 6.2$ expression in V2a INs, but indirectly downregulate its expression in MNs via a repression mechanism that remains to be identified. Identification of the OC factor binding sites in the regulatory sequences of $N k x 6.2$ could address these possibilites. However, $N k x 6.2$ regulatory sequences remain to be characterized and potential OC factor binding sites in the genome are common. Therefore, ChIP-seq experiments on isolated spinal neuron subsets would be necessary to identify the genes directly regulated by OC factors in specific spinal populations. Potential OC factor targets that could mediate indirect repressive regulations include microRNAs (miRs), as OC factors have been shown to stimulate miR-122 in hepatic cells ${ }^{49}$. However, miR contributions to spinal cord development remain poorly described. Third, OC factors may associate with cell-type specific cofactors or transcriptional complexes to differentially regulate expression in distinct cell populations. In hepatic cells, OC factors interact with the CBP, p300 and PGC- 1 cofactors to stimulate the expression of specific target genes ${ }^{48,50}$. Similarly, we recently demonstrated that CBP and p300 cofactors contribute to the regulation of Isl 1 expression by OC factors ${ }^{51}$. However, association of OC factors with transcriptional repressors remains to be demonstrated. This highlights the urgent need to identify direct transcriptional targets and molecular partners of OC factors in specific spinal neuron populations to better understand their contribution to spinal cord development.

Inactivation of the Nkx6.2 gene in mice or mutations within the orthologous gene in humans result respectively in motor deficits that have been attributed previously to defective central myelination ${ }^{23,52,53}$. Although we cannot formally exclude contributions from such myelin-associated pathophysiology, perturbed embryonic expression of Nkx6.2 in V2a INs and in $\mathrm{LMCl}$ MNs, which are part of spinal motor circuits including the locomotor network, prompted us to evaluate the effects of the absence of Nkx6.2 specifically on locomotor behavior in Nkx6.2-null adults and neonates, and on FL in neonates. Adult locomotion showed increased gait frequency and altered paw-substrate contact patterns, and neonatal locomotion (swimming) showed increased cycle frequency and defective alternation, suggesting potential perturbations of the locomotor network. Recording of FL in isolated neonatal $N k x 6.2$-null spinal cord preparations revealed no obvious perturbation of pattern, but a change in the frequency profile characterized by a shift from the control bimodal frequency distribution to a distribution dominated by a single intermediate frequency mode. In the locomotor CPG, V2a INs provide left/ right alternation at high frequency ${ }^{54}$ whereas their target V0 INs are divided into inhibitory or excitatory subsets that ensure left/right alternation at low or high locomotor speeds, respectively ${ }^{55}$. Since we find that the absence of Nkx6.2 perturbs the development of $\mathrm{V} 2 \mathrm{a}$ INs, it is possible that this also perturbs their connectivity in a way that alters locomotor network output. Our observations in the current study are unlikely to be secondary to the absence of $N k x 6.2$ expression in oligodendrocytes, even though myelination of cervical spinal MN axons and central vestibular projections commences perinatally in mice ${ }^{41,56}$. Comparison of the locomotor defects in $N k x 6.2$ and in constitutive or MN-specific conditional $O c$ mutant animals unfortunately can not be conducted because constitutive ${ }^{24}$ and conditional (data not shown) Oc mutants die at birth for reasons that remain to be determined. Similarly, identifying the reasons why the RF portion of the adult quadriceps of $N k x 6.2$-null adults shows an increase in the number of fatiguable large diameter fibers at the expense of fatigue-resistant small diameter fibers will also require further investigation. 
In spinal neuronal progenitors, Nkx6.2 function overlaps with that of $\mathrm{Nkx} 6.1$ when it comes to ventral patterning of the spinal cord ${ }^{35}$. However, Nkx6.2 is additionally expressed later in V2a INs and in a pool of LMCl MNs (ref. ${ }^{38}$ and the present study). Since the only progenitors that express $N k x 6.2$ are the V1 progenitors ${ }^{37}$, expression in the postmitotic V2a INs and $\mathrm{LMCl}$ MNs is not merely a carry-over from progenitor expression but rather a secondarily regulated pattern. That Nkx6.2 is actively regulated in two postmitotic spinal neuron populations suggests a possible role in controlling their developmental programs. Our phenotypic study of Nkx6.2-null embryos suggests that Nkx6.2 regulates distinct biological processes in MNs and ventral INs. In Nkx6.2-null embryos, the number of $\mathrm{LMCl}$ MNs was normal at e12.5 but depleted at e14.5, indicating that Nkx6.2 is required for the survival of some LMCl MNs. The most immediate explanation is that the missing MNs correspond to the RF motor pool that normally expresses $N k x 6.2$. However, several observations argue against this simple interpretation. First, $\beta$-galactosidase-positive MNs, i.e. the MNs in which the Nkx6.2 regulating sequences are normally activated, are found in the ventral portion of the $\mathrm{LMCl}$ in $N k x 6.2$-null embryos, in an area that does not contain RF MNs. Second, motor projections towards the RF are present and seem unaltered in the $N k x 6.2$ mutant $^{38}$. Third, the density of neuromuscular junctions in the RF is normal in the Nkx6.2 mutant (data not shown). Taken together, these data suggest that RF MNs are not significantly affected by the absence of Nkx6.2. In an attempt to identify the MN subset that could be missing in the $N k x 6.2$ mutant, we evaluated the size of the Nkx6.1 + or Er81 + motor pools but found that these MNs were also present in normal numbers. Thus, the identity of the $\mathrm{LMCl}$ cells that are missing in Nkx6.2 mutants and the apparently non-cell autonomous mechanisms whereby Nkx6.2 promotes their survival remain to be determined.

In contrast to $\mathrm{LMCl} \mathrm{MNs}$, the number of $\mathrm{V} 2 \mathrm{a}$ INs is largely unaffected by the absence of Nkx6.2, except for a modest reduction in the thoracic spinal cord. However, the spatial distribution of the V2a INs was abnormal. This is consistent with the downregulation of $N k x 6.2$ expression in V2a INs and the perturbation of V2a IN distribution recently reported in constitutive $O c$ mutant embryos ${ }^{13}$. These data suggest that $\mathrm{Nkx} 6.2$ contributes to regulate V2a IN distribution downstream of the OC factors. To our knowledge, Nkx6.2 has not previously been reported to regulate neuronal localization, and the mechanisms whereby it does here are not known. Similarly, OC factors and Pou $2 \mathrm{f} 2$ have recently been shown to regulate the distribution of V2a and V2b INs but their downstream targets in this process remain unknown. A genome-wide systematic study of the transcriptional targets of the three OC transcription factors would be required to understand the downstream genetic programs and mechanisms regulating the distribution of ventral INs during spinal cord development.

\section{Data availability}

All data generated or analysed during this study are included in this published article (and its Supplementary Information Files) or available upon request.

Received: 6 September 2019; Accepted: 9 January 2020;

Published online: 22 January 2020

\section{References}

1. Lu, D. C., Niu, T. \& Alaynick, W. A. Molecular and cellular development of spinal cord locomotor circuitry. Front. Mol. Neurosci. 8, 25, https://doi.org/10.3389/fnmol.2015.00025 (2015).

2. Jessell, T. M. Neuronal specification in the spinal cord: inductive signals and transcriptional codes. Nat. Rev. Genet. 1, 20-29, https:// doi.org/10.1038/35049541 (2000).

3. Landmesser, L. The distribution of motoneurones supplying chick hind limb muscles. J. Physiol. 284, 371-389 (1978).

4. Landmesser, L. The development of motor projection patterns in the chick hind limb. J. Physiol. 284, 391-414 (1978).

5. Jacquemin, P., Lannoy, V. J., Rousseau, G. G. \& Lemaigre, F. P. OC-2, a novel mammalian member of the ONECUT class of homeodomain transcription factors whose function in liver partially overlaps with that of hepatocyte nuclear factor-6. J. Biol. Chem. 274, 2665-2671 (1999).

6. Jacquemin, P. et al. Cloning and embryonic expression pattern of the mouse Onecut transcription factor OC-2. Gene Expr Patterns 3, 639-644, doi:S1567133X03001108 (2003)

7. Vanhorenbeeck, V., Jacquemin, P., Lemaigre, F. P. \& Rousseau, G. G. OC-3, a novel mammalian member of the ONECUT class of transcription factors. Biochem. Biophys. Res. Commun. 292, 848-854, https://doi.org/10.1006/bbrc.2002.6760 (2002).

8. Landry, C. et al. HNF-6 is expressed in endoderm derivatives and nervous system of the mouse embryo and participates to the crossregulatory network of liver-enriched transcription factors. Dev. Biol. 192, 247-257, https://doi.org/10.1006/dbio.1997.8757 (1997).

9. Lemaigre, F. P. et al. Hepatocyte nuclear factor 6, a transcription factor that contains a novel type of homeodomain and a single cut domain. Proc. Natl Acad. Sci. USA 93, 9460-9464 (1996).

10. Espana, A. \& Clotman, F. Onecut factors control development of the Locus Coeruleus and of the mesencephalic trigeminal nucleus. Mol. Cell Neurosci. 50, 93-102, https://doi.org/10.1016/j.mcn.2012.04.002 (2012).

11. Roy, A. et al. Onecut transcription factors act upstream of Isl1 to regulate spinal motoneuron diversification. Dev. 139, 3109-3119, https://doi.org/10.1242/dev.078501 (2012).

12. Kabayiza, K. U. et al. The Onecut Transcription Factors Regulate Differentiation and Distribution of Dorsal Interneurons during Spinal Cord Development. Front. Mol. Neurosci. 10, 157, https://doi.org/10.3389/fnmol.2017.00157 (2017).

13. Harris, A. et al. Onecut Factors and Pou2f2 Regulate the Distribution of V2 Interneurons in the Mouse Developing Spinal Cord. Front. Cell Neurosci. 13, 184, https://doi.org/10.3389/fncel.2019.00184 (2019).

14. Audouard, E. et al. The Onecut transcription factor HNF-6 contributes to proper reorganization of Purkinje cells during postnatal cerebellum development. Mol. Cell Neurosci. 56, 159-168, https://doi.org/10.1016/j.mcn.2013.05.001 (2013).

15. Espana, A. \& Clotman, F. Onecut transcription factors are required for the second phase of development of the A13 dopaminergic nucleus in the mouse. J. Comp. Neurol. 520, 1424-1441, https://doi.org/10.1002/cne.22803 (2012).

16. Stam, F. J. et al. Renshaw cell interneuron specialization is controlled by a temporally restricted transcription factor program. Dev. 139, 179-190, https://doi.org/10.1242/dev.071134 (2012).

17. Audouard, E. et al. The Onecut transcription factor HNF-6 regulates in motor neurons the formation of the neuromuscular junctions. PLoS One 7, e50509, https://doi.org/10.1371/journal.pone.0050509 (2012).

18. Francius, C. \& Clotman, F. Dynamic expression of the Onecut transcription factors HNF-6, OC-2 and OC-3 during spinal motor neuron development. Neurosci. 165, 116-129, https://doi.org/10.1016/j.neuroscience.2009.09.076 (2010). 
19. Rhee, H. S. et al. Expression of Terminal Effector Genes in Mammalian Neurons Is Maintained by a Dynamic Relay of Transient Enhancers. Neuron 92, 1252-1265, https://doi.org/10.1016/j.neuron.2016.11.037 (2016).

20. Velasco, S. et al. A Multi-step Transcriptional and Chromatin State Cascade Underlies Motor Neuron Programming from Embryonic Stem Cells. Cell Stem Cell 20, 205-217 e208, https://doi.org/10.1016/j.stem.2016.11.006 (2017).

21. Wilzen, A. et al. The Phox2 pathway is differentially expressed in neuroblastoma tumors, but no mutations were found in the candidate tumor suppressor gene PHOX2A. Int. J. Oncol. 34, 697-705 (2009).

22. Dessaud, E. et al. Interpretation of the sonic hedgehog morphogen gradient by a temporal adaptation mechanism. Nature 450, 717720, doi:nature06347 (2007).

23. Southwood, C. et al. CNS myelin paranodes require Nkx6-2 homeoprotein transcriptional activity for normal structure. J Neurosci 24, 11215-11225, doi:24/50/11215 (2004).

24. Clotman, F. et al. Control of liver cell fate decision by a gradient of TGF beta signaling modulated by Onecut transcription factors. Genes. Dev. 19, 1849-1854, https://doi.org/10.1101/gad.340305 (2005).

25. Palmesino, E. et al. Foxp1 and lhx1 coordinate motor neuron migration with axon trajectory choice by gating Reelin signalling. PLoS Biol. 8, e1000446, https://doi.org/10.1371/journal.pbio.1000446 (2010).

26. Wang, W. et al. Sinomenine Attenuates Angiotensin II-Induced Autophagy via Inhibition of P47-Phox Translocation to the Membrane and Influences Reactive Oxygen Species Generation in Podocytes. Kidney Blood Press. Res. 41, 158-167, https://doi. org/10.1159/000443417 (2016).

27. Di Bonito, M. et al. Loss of Projections, Functional Compensation, and Residual Deficits in the Mammalian Vestibulospinal System of Hoxb1-Deficient Mice. eNeuro 2, doi:10.1523/ENEURO.0096-15.2015 (2015).

28. Zuchner, M., Kondratskaya, E., Sylte, C. B., Glover, J. C. \& Boulland, J. L. Rapid recovery and altered neurochemical dependence of locomotor central pattern generation following lumbar neonatal spinal cord injury. J. Physiol. 596, 281-303, https://doi.org/10.1113/ JP274484 (2018).

29. Kondratskaya, E. et al. Locomotor central pattern generator excitability states and serotonin sensitivity after spontaneous recovery from a neonatal lumbar spinal cord injury. Brain Res 1708, 10-19, doi:S0006-8993(18)30611-5 (2019).

30. Kjaerulff, O. \& Kiehn, O. Distribution of networks generating and coordinating locomotor activity in the neonatal rat spinal cord in vitro: a lesion study. J. Neurosci. 16, 5777-5794 (1996).

31. Whelan, P., Bonnot, A. \& O'Donovan, M. J. Properties of rhythmic activity generated by the isolated spinal cord of the neonatal mouse. J. Neurophysiol. 84, 2821-2833, https://doi.org/10.1152/jn.2000.84.6.2821 (2000).

32. Hinckley, C. A., Hartley, R., Wu, L., Todd, A. \& Ziskind-Conhaim, L. Locomotor-like rhythms in a genetically distinct cluster of interneurons in the mammalian spinal cord. J Neurophysiol 93, 1439-1449, doi:00647.2004 (2005).

33. Jiang, Z., Carlin, K. P. \& Brownstone, R. M. An in vitro functionally mature mouse spinal cord preparation for the study of spinal motor networks. Brain Res 816, 493-499, doi:S0006-8993(98)01199-8 (1999).

34. Chen, J. A. et al. Mir-17-3p controls spinal neural progenitor patterning by regulating Olig2/Irx3 cross-repressive loop. Neuron 69, 721-735, doi:S0896-6273(11)00062-6 (2011).

35. Vallstedt, A. et al. Different levels of repressor activity assign redundant and specific roles to Nkx6 genes in motor neuron and interneuron specification. Neuron 31, 743-755, doi:S0896-6273(01)00412-3 (2001).

36. Sander, M. et al. Ventral neural patterning by Nkx homeobox genes: Nkx6.1 controls somatic motor neuron and ventral interneuron fates. Genes. Dev. 14, 2134-2139 (2000).

37. Cai, J. et al. Expression and regulation of the chicken Nkx-6.2 homeobox gene suggest its possible involvement in the ventral neural patterning and cell fate specification. Dev Dyn 216, 459-468, doi:10.1002/(SICI) 1097-0177(199912)216:4/5<459::AIDDVDY14>3.0.CO;2-7 (1999).

38. De Marco Garcia, N. V. \& Jessell, T. M. Early motor neuron pool identity and muscle nerve trajectory defined by postmitotic restrictions in Nkx6.1 activity. Neuron 57, 217-231, doi:S0896-6273(07)01023-9 (2008).

39. Sockanathan, S. \& Jessell, T. M. Motor neuron-derived retinoid signaling specifies the subtype identity of spinal motor neurons. Cell 94, 503-514, doi:S0092-8674(00)81591-3 (1998).

40. Sockanathan, S., Perlmann, T. \& Jessell, T. M. Retinoid receptor signaling in postmitotic motor neurons regulates rostrocaudal positional identity and axonal projection pattern. Neuron 40, 97-111, doi:S0896627303005324 (2003).

41. Gow, A., Friedrich, V. L. Jr. \& Lazzarini, R. A. Myelin basic protein gene contains separate enhancers for oligodendrocyte and Schwann cell expression. J. Cell Biol. 119, 605-616, https://doi.org/10.1083/jcb.119.3.605 (1992).

42. Ribeiro, A. F. Jr. et al. Muscle satellite cells and impaired late stage regeneration in different murine models for muscular dystrophies. Sci. Rep. 9, 11842, https://doi.org/10.1038/s41598-019-48156-7 (2019).

43. Cai, J. et al. Mice lacking the Nkx6.2 (Gtx) homeodomain transcription factor develop and reproduce normally. Mol. Cell Biol. 21, 4399-4403, https://doi.org/10.1128/MCB.21.13.4399-4403.2001 (2001).

44. Lin, J. H. et al. Functionally related motor neuron pool and muscle sensory afferent subtypes defined by coordinate ETS gene expression. Cell 95, 393-407, doi:S0092-8674(00)81770-5 (1998).

45. Clotman, F. et al. The onecut transcription factor HNF6 is required for normal development of the biliary tract. Dev. 129, 1819-1828 (2002).

46. Jacquemin, P. et al. Transcription factor hepatocyte nuclear factor 6 regulates pancreatic endocrine cell differentiation and controls expression of the proendocrine gene ngn3. Mol. Cell Biol. 20, 4445-4454 (2000).

47. Jacquemin, P., Lemaigre, F. P. \& Rousseau, G. G. The Onecut transcription factor HNF-6 (OC-1) is required for timely specification of the pancreas and acts upstream of Pdx-1 in the specification cascade. Dev Biol 258, 105-116, doi:S0012160603001155 (2003).

48. Lannoy, V. J., Rodolosse, A., Pierreux, C. E., Rousseau, G. G. \& Lemaigre, F. P. Transcriptional stimulation by hepatocyte nuclear factor-6. Target-specific recruitment of either CREB-binding protein (CBP) or p300/CBP-associated factor (p/CAF). J. Biol. Chem. 275, 22098-22103, https://doi.org/10.1074/jbc.M000855200 (2000).

49. Laudadio, I. et al. A feedback loop between the liver-enriched transcription factor network and miR-122 controls hepatocyte differentiation. Gastroenterology 142, 119-129, https://doi.org/10.1053/j.gastro.2011.09.001 (2012).

50. Beaudry, J. B. et al. Threshold levels of hepatocyte nuclear factor 6 (HNF-6) acting in synergy with HNF-4 and PGC-1alpha are required for time-specific gene expression during liver development. Mol Cell Biol 26, 6037-6046, doi:26/16/6037 (2006).

51. Toch, M. \& Clotman, F. CBP and p300 coactivators contribute to the maintenance of Isll expression by the Onecut transcription factors in embryonic spinal motor neurons. Mol. Cell Neurosci. 101, 103411, https://doi.org/10.1016/j.mcn.2019.103411 (2019).

52. Chelban, V. et al. Mutations in NKX6-2 Cause Progressive Spastic Ataxia and Hypomyelination. Am J Hum Genet 100, 969-977, doi:S0002-9297(17)30196-9 (2017).

53. Chelban, V., Kaya, N., Alkuraya, F. \& Houlden, H. NKX6-2-Related Disorder. doi:NBK531509 [bookaccession] (1993).

54. Crone, S. A. et al. Genetic ablation of V2a ipsilateral interneurons disrupts left-right locomotor coordination in mammalian spinal cord. Neuron 60, 70-83, doi:S0896-6273(08)00677-6 (2008).

55. Talpalar, A. E. et al. Dual-mode operation of neuronal networks involved in left-right alternation. Nat. 500, 85-88, https://doi. org/10.1038/nature12286 (2013).

56. Lorente De, N. R. Anatomy of the eighth nerve. Cent. projection nerve End. Intern. ear. Laryngoscope 43, 1-38 (1933). 


\section{Acknowledgements}

We thank members of the NEDI lab for material, technical support and discussions. This work was supported by grants from the "Fonds spéciaux de recherche" (FSR) of the Universite catholique de Louvain, by the "Actions de Recherche Concertées (ARC)" \#17/22-079 of the "Direction générale de l'Enseignement non obligatoire et de la Recherche scientifique - Direction de la Recherche scientifique - Communauté française de Belgique" and granted by the "Académie universitaire 'Louvain", by a "Projet de recherche (PDR)" \#T.0117.13 and an "Equipement (EQP)" funding \#U.N027.14 of the Fonds de la Recherche Scientifique (F.R.S.-FNRS) and by the Association Belge contre les Maladies neuro-Musculaires (ABMM, Belgium) to F.C., by South-East Norway Health Authority grant no. 2014119 to J.-L.B., by Norwegian Research Council grant no. 230000/F20 and EUfunded ERA-NET Neuron grant (CERMOD) to J.C.G., by a grant (R01EY029705) from the National Institutes of Health/National Eye Institute to X.M. and by NINDS in the National Institutes of Health, grant no. NS102678 to A.G. A.H., S.D. and C.B. held FRIA grants and M.H.-F. held a Research Associate grant from the F.R.S.-FNRS. F.C. and F.T. are Senior Research Associate and Research Director of the F.R.S.-FNRS, respectively.

\section{Author contributions}

M.T., A.H., E.K., J.-L.B., J.C.G., N.D., F.T. and F.C. designed the experiments. M.H.-F., X.M. and A.G. provided mice and contributed to initial discussions. M.T., A.H., O.S., E.K., J.-L.B., N.D., S.D. and C.B. performed the experiments, M.T., A.H., O.S., E.K., and J.-L.B. contributed to data analyses and all the authors discussed the data for interpretation. M.T. and F.C. wrote the initial draft of the manuscript, and revisions were made by J.C.G., J.-L.B., E.K., A.G., M.T. and F.C.

\section{Competing interests}

The authors declare no competing interests.

\section{Additional information Supplementary information is available for this paper at https://doi.org/10.1038/s41598-020-57945-4.}

Correspondence and requests for materials should be addressed to F.C.

Reprints and permissions information is available at www.nature.com/reprints.

Publisher's note Springer Nature remains neutral with regard to jurisdictional claims in published maps and institutional affiliations.

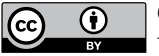

Open Access This article is licensed under a Creative Commons Attribution 4.0 International License, which permits use, sharing, adaptation, distribution and reproduction in any medium or format, as long as you give appropriate credit to the original author(s) and the source, provide a link to the Creative Commons license, and indicate if changes were made. The images or other third party material in this article are included in the article's Creative Commons license, unless indicated otherwise in a credit line to the material. If material is not included in the article's Creative Commons license and your intended use is not permitted by statutory regulation or exceeds the permitted use, you will need to obtain permission directly from the copyright holder. To view a copy of this license, visit http://creativecommons.org/licenses/by/4.0/.

(C) The Author(s) 2020 